\title{
HOMOTOPICALLY EQUIVALENT SIMPLE LOOPS ON 2-BRIDGE SPHERES IN 2-BRIDGE LINK COMPLEMENTS
} (I)

\author{
DONGHI LEE AND MAKOTO SAKUMA
}

\begin{abstract}
In this paper and its two sequels, we give a necessary and sufficient condition for two essential simple loops on a 2-bridge sphere in a 2-bridge link complement to be homotopic in the link complement. This paper treats the case when the 2-bridge link is a $(2, p)$-torus link, where more cases of homotopy arise, and its sequels will treat the remaining cases.
\end{abstract}

\section{INTRODUCTION}

Let $K$ be a 2-bridge link in $S^{3}$ and let $S$ be a 4-punctured sphere in $S^{3}-K$ obtained from a 2-bridge sphere of $K$. In [6], we gave a complete characterization of those essential simple loops in $S$ which are null-homotopic in $S^{3}-K$, and by using the result, we described all upper-meridian-pair-preserving epimorphisms between 2-bridge link groups. The purpose of this paper and its sequels is to give a necessary and sufficient condition for two essential simple loops on $S$ to be homotopic in $S^{3}-K$.

It has been proved by Weinbaum [16] and Appel and Schupp [1] that the word and conjugacy problems for prime alternating link groups are solvable, by using small cancellation theory (see also [3] and references in it). Moreover, it was also shown by Sela [15] and Préaux [12] that the word and conjugacy problems for any link group are solvable. A characteristic feature of this series of papers including [6] is that we give a complete answer to special (but also natural) word and conjugacy problems for the groups of 2-bridge links, which form a special (but also important) family of prime alternating links. The key tool used in the proofs is small cancellation theory, applied to two-generator and one-relator presentations of 2-bridge link groups.

2010 Mathematics Subject Classification. Primary 20F06, 57M25

The first author was supported by Basic Science Research Program through the National Research Foundation of Korea(NRF) funded by the Ministry of Education, Science and Technology(2009-0065798). The second author was supported by JSPS Grants-in-Aid 22340013 and 21654011. 
In this paper, we treat the case when the 2-bridge link is a $(2, p)$-torus link (Main Theorem 2.7), where more cases of homotopy arise. The remaining cases will be treated in the sequels of this paper [7] and [8], and these results will be used in [9] to show the existence of a variation of McShane's identity for 2-bridge links. For an overview of this series of works, we refer the reader to the research announcement [5].

This paper is organized as follows. In Section 2, we recall basic facts concerning 2-bridge links, and describe the main result of this paper. In Section 3 , we recall the upper presentation of a 2-bridge link group, and recall key facts established in [6] concerning the upper presentation. These facts are to be used throughout this series of papers. In Section 4 , we establish a very strong structure theorem (Theorems 4.9 and 4.11) for the annular diagram which arises in the study of the conjugacy problem by using small cancellation theory. Finally, Section 5 is devoted to the proof of the main result.

\section{MAIN RESULT}

Consider the discrete group, $H$, of isometries of the Euclidean plane $\mathbb{R}^{2}$ generated by the $\pi$-rotations around the points in the lattice $\mathbb{Z}^{2}$. Set $\left(\boldsymbol{S}^{2}, \boldsymbol{P}\right):=$ $\left(\mathbb{R}^{2}, \mathbb{Z}^{2}\right) / H$ and call it the Conway sphere. Then $\boldsymbol{S}^{2}$ is homeomorphic to the 2-sphere, and $\boldsymbol{P}$ consists of four points in $\boldsymbol{S}^{2}$. We also call $\boldsymbol{S}^{2}$ the Conway sphere. Let $\boldsymbol{S}:=\boldsymbol{S}^{2}-\boldsymbol{P}$ be the complementary 4-times punctured sphere. For each $r \in \hat{\mathbb{Q}}:=\mathbb{Q} \cup\{\infty\}$, let $\alpha_{r}$ be the unoriented simple loop in $\boldsymbol{S}$ obtained as the projection of any straight line in $\mathbb{R}^{2}-\mathbb{Z}^{2}$ of slope $r$. Then $\alpha_{r}$ is essential in $\boldsymbol{S}$, i.e., it does not bound a disk nor a once-punctured disk in $\boldsymbol{S}$. Conversely, any essential simple loop in $\boldsymbol{S}$ is isotopic to $\alpha_{r}$ for a unique $r \in \hat{\mathbb{Q}}$. Then $r$ is called the slope of the simple loop. Similarly, any simple arc $\delta$ in $\boldsymbol{S}^{2}$ joining two different points in $\boldsymbol{P}$ such that $\delta \cap \boldsymbol{P}=\partial \delta$ is isotopic to the image of a line in $\mathbb{R}^{2}$ of some slope $r \in \hat{\mathbb{Q}}$ which intersects $\mathbb{Z}^{2}$. We call $r$ the slope of $\delta$. Thus, for every slope $r \in \hat{\mathbb{Q}}$, there exist two arcs and one loop of slope $r$ in $\left(\boldsymbol{S}^{2}, \boldsymbol{P}\right)$ (all unoriented).

A trivial tangle is a pair $\left(B^{3}, t\right)$, where $B^{3}$ is a 3 -ball and $t$ is a union of two arcs properly embedded in $B^{3}$ which is parallel to a union of two mutually disjoint arcs in $\partial B^{3}$. By a rational tangle, we mean a trivial tangle $\left(B^{3}, t\right)$ which is endowed with a homeomorphism from $\left(\partial B^{3}, \partial t\right)$ to $\left(\boldsymbol{S}^{2}, \boldsymbol{P}\right)$. Through the homeomorphism we identify the boundary of a rational tangle with the Conway sphere. Thus the slope of an essential simple loop in $\partial B^{3}-t$ is defined. We define the slope of a rational tangle to be the slope of an essential loop on $\partial B^{3}-t$ which bounds a disk in $B^{3}$ separating the components of $t$. (Such a 
loop is unique up to isotopy on $\partial B^{3}-t$ and is called a meridian of the rational tangle.) We denote a rational tangle of slope $r$ by $\left(B^{3}, t(r)\right)$. By van Kampen's theorem, the fundamental group $\pi_{1}\left(B^{3}-t(r)\right)$ is identified with the quotient $\pi_{1}(\boldsymbol{S}) /\left\langle\left\langle\alpha_{r}\right\rangle\right\rangle$, where $\left\langle\left\langle\alpha_{r}\right\rangle\right\rangle$ denotes the normal closure.

For each $r \in \hat{\mathbb{Q}}$, the 2-bridge link $K(r)$ of slope $r$ is defined to be the sum of the rational tangles of slopes $\infty$ and $r$, namely, $\left(S^{3}, K(r)\right)$ is obtained from $\left(B^{3}, t(\infty)\right)$ and $\left(B^{3}, t(r)\right)$ by identifying their boundaries through the identity map on the Conway sphere $\left(\boldsymbol{S}^{2}, \boldsymbol{P}\right)$. (Recall that the boundaries of rational tangles are identified with the Conway sphere.) By van Kampen's theorem again, the link group $G(K(r)):=\pi_{1}\left(S^{3}-K(r)\right)$ is identified with $\pi_{1}(\boldsymbol{S}) /\left\langle\left\langle\alpha_{\infty}, \alpha_{r}\right\rangle\right\rangle$. Note that $K(r)$ has one or two components according as the denominator of $r$ is odd or even. We call $\left(B^{3}, t(\infty)\right)$ and $\left(B^{3}, t(r)\right)$, respectively, the upper tangle and lower tangle of the 2-bridge link. Also note that $\left(S^{3}, K(r)\right)$ is homeomorphic to $\left(S^{3}, K(r+1)\right)$, so that $r$ matters only modulo 1 .

Let $\mathcal{D}$ be the Farey tessellation, that is, the tessellation of the upper half plane $\mathbb{H}^{2}$ by ideal triangles which are obtained from the ideal triangle with the ideal vertices $0,1, \infty \in \hat{\mathbb{Q}}$ by repeated reflection in the edges. Then $\hat{\mathbb{Q}}$ is identified with the set of the ideal vertices of $\mathcal{D}$. For each $r \in \hat{\mathbb{Q}}$, let $\Gamma_{r}$ be the group of automorphisms of $\mathcal{D}$ generated by reflections in the edges of $\mathcal{D}$ with an endpoint $r$. It should be noted that $\Gamma_{r}$ is isomorphic to the infinite dihedral group and the region bounded by two adjacent edges of $\mathcal{D}$ with an endpoint $r$ is a fundamental domain for the action of $\Gamma_{r}$ on $\mathbb{H}^{2}$, by virtue of Poincaré's fundamental polyhedron theorem (see, for example, [13]). Let $\hat{\Gamma}_{r}$ be the group generated by $\Gamma_{r}$ and $\Gamma_{\infty}$. When $r \in \mathbb{Q}-\mathbb{Z}, \hat{\Gamma}_{r}$ is equal to the free product $\Gamma_{r} * \Gamma_{\infty}$, having a fundamental domain shown in Figure 1. Otherwise, $\hat{\Gamma}_{r}$ is the group generated by the reflections in the edges of $\mathcal{D}$ or $\Gamma_{\infty}$ according as $r \in \mathbb{Z}$ or $r=\infty$.

The following key observation was made in [1].

Proposition 2.1. [11, Proposition 4.6] Let $r \in \hat{\mathbb{Q}}$. If two elements $s$ and $s^{\prime}$ of $\hat{\mathbb{Q}}$ belong to the same $\hat{\Gamma}_{r}$-orbit, then the unoriented loops $\alpha_{s}$ and $\alpha_{s^{\prime}}$ are homotopic in $S^{3}-K(r)$.

Proof. We give a proof to this proposition from a view point brought to the authors by the referee. Let $\gamma_{0} \in \Gamma_{\infty}$ be the reflection of $\mathcal{D}$ in the Farey edge $\langle\infty, 0\rangle$. Note that $\gamma_{0}$ is induced by a self-homeomorphism, $\tilde{\gamma}_{0}$, of $\boldsymbol{S}$ which is a reflection in the simple loop $\alpha_{0}$ of slope 0 . Recall that $\boldsymbol{S}$ is regarded as a subspace of $S^{3}-K(r)$, and denote by $j: S \rightarrow S^{3}-K(r)$ the inclusion map. Then $j$ can be homotoped to $j \circ \tilde{\gamma}_{0}$ by swapping the upper and lower 


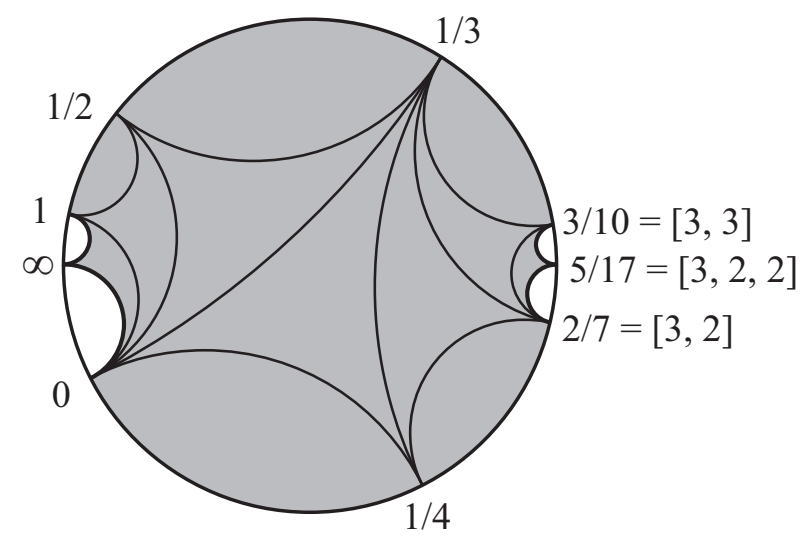

Figure 1. A fundamental domain of $\hat{\Gamma}_{r}$ in the Farey tessellation (the shaded domain) for $r=5 / 17=\frac{1}{3+\frac{1}{2+\frac{1}{2}}}=:[3,2,2]$

hemispheres of $\boldsymbol{S}$ bounded $\alpha_{0}$ through the tangle $\left(B^{3}, t(\infty)\right)$, as in Figure 3(a). Hence $\alpha_{\gamma_{0}(s)}=j \circ \tilde{\gamma}_{0}\left(\alpha_{s}\right)$ is homotopic to $j\left(\alpha_{s}\right)=\alpha_{s}$ in $S^{3}-K(r)$.

Let $\gamma_{1} \in \Gamma_{\infty}$ be the reflection of $\mathcal{D}$ in the Farey edge $\langle\infty, 1\rangle$, and set $\varphi=$ $\gamma_{1} \gamma_{0} \in \Gamma_{\infty}$. Then $\varphi$ is the parabolic transformation of $\mathcal{D}$, centered on $\infty$, by two units in the counter-clockwise direction, and it is induced by a selfhomeomorphism $\tilde{\varphi}$ of $\boldsymbol{S}$ which is a Dehn twist along the simple loop $\alpha_{\infty}$. Since $\alpha_{\infty}$ bounds a disk in $B^{3}-t(\infty), j$ can be homotoped to $j \circ \tilde{\varphi}$ by a homotopy in $B^{3}-t(\infty)$. Hence $\varphi$ also preserves the homotopy classes, in $S^{3}-K(r)$, of simple loops in $\boldsymbol{S}$.

Since $\Gamma_{\infty}$ is generated by $\gamma_{0}$ and $\varphi$, the above observations imply that $\Gamma_{\infty}$ preserves the homotopy classes, in $S^{3}-K(r)$, of simple loops in $\boldsymbol{S}$. We can also see that $\Gamma_{r}$ has the same property by using homotopies in $B^{3}-t(r)$. Hence we obtain the desired result.

Thus the following question naturally arises.

Question 2.2. Is the converse to the above proposition valid? If not, when are two essential simple loops on a 2-bridge sphere in a 2-bridge link complement homotopic in the link complement?

If $r=\infty$, then $G(K(\infty))$ is a rank 2 free group, and the result of Komori and Series [4, Theorem 1.2] is equivalent to the affirmative answer to the first question in the above. On the other hand, if $r=0$ (or any integer), then 
$G(K(0))$ is the infinite cyclic group, and $\hat{\Gamma}_{0}$ is equal to the group generated by the reflections in the edges of any of $\mathcal{D}$. In particular, any Farey triangle is a fundamental domain for the action of $\hat{\Gamma}_{0}$ on $\mathbb{H}^{2}$. Hence, any $s \in \hat{\mathbb{Q}}$ belongs to the $\hat{\Gamma}_{0^{-}}$orbit of one and only one of $\{0,1, \infty\}$. But since $\alpha_{1}$ is not nullhomotopic while $\alpha_{0}$ and $\alpha_{\infty}$ are null-homotopic in $S^{3}-K(0)$, every simple loop which is not null-homotopic has slope belonging to the $\hat{\Gamma}_{0}$-orbit of 1 . This yields the affirmative answer to the first question in the above for $r=0$. In [6], we gave the following complete characterization of those essential simple loops on 2-bridge spheres of 2-bridge links which are null-homotopic in the link complements.

Theorem 2.3. [6, Main Theorem 2.3] Let $r \in \hat{\mathbb{Q}}$. The loop $\alpha_{s}$ is nullhomotopic in $S^{3}-K(r)$ if and only if $s$ belongs to the $\hat{\Gamma}_{r}$-orbit of $\infty$ or $r$.

The purpose of this paper and its sequels is to solve the above question. In this paper, we give an answer to the question for the $(2, p)$-torus link $K(1 / p)$ with $p \geq 2$ (Main Theorem 2.7).

In order to state the main result, we introduce some notation. For a rational number $r=1 / p$, let $\tau$ be the automorphism of the Farey tessellation defined as follows:

(i) If $p=2 p_{0}$ for some $p_{0} \in \mathbb{Z}_{+}$, then $\tau$ is the reflection in the Farey edge $\left\langle 0,1 / p_{0}\right\rangle$.

(ii) If $p=2 p_{0}+1$ for some $p_{0} \in \mathbb{Z}_{+}$, then $\tau$ is the reflection in the geodesic with an endpoint 0 which bisects the Farey edge $\left\langle 1 / p_{0}, 1 /\left(p_{0}+1\right)\right\rangle$. Thus $\tau$ is the reflection in the geodesic with endpoints 0 and $2 /\left(2 p_{0}+1\right)$.

In both cases, $\tau$ is the reflection in the geodesic with endpoints 0 and $2 / p$, and interchanges $\infty$ with $1 / p$ (see Figure 2). Moreover, we can see that the action of $\tau$ on the vertex set $\hat{\mathbb{Q}}$ of the Farey tessellation is given by $\tau(c / d)=c /(c p-d)$. In particular, if $\tau\left(q_{1} / p_{1}\right)=q_{2} / p_{2}$, where $\left(p_{i}, q_{i}\right)$ is a pair of relatively prime positive integers, then $q_{1}=q_{2}$ and $q_{1} /\left(p_{1}+p_{2}\right)=1 / p$.

Set $\tilde{\Gamma}_{1 / p}=\left\langle\hat{\Gamma}_{1 / p}, \tau\right\rangle$, the group generated by $\hat{\Gamma}_{1 / p}$ and $\tau$. Then $\tilde{\Gamma}_{1 / p}$ is a $\mathbb{Z}_{2}$-extension of $\hat{\Gamma}_{1 / p}$. Now our main theorem is stated as follows.

Main Theorem 2.4. Let $p \geq 2$ be an integer. Then, for two distinct $s, s^{\prime} \in \hat{\mathbb{Q}}$, the unoriented loops $\alpha_{s}$ and $\alpha_{s^{\prime}}$ are homotopic in $S^{3}-K(1 / p)$ if and only if $s$ and $s^{\prime}$ lie in the same $\tilde{\Gamma}_{1 / p}$-orbit.

The if part of Main Theorem 2.4 can be easily proved by the following nice observation pointed out by the referee. By virtue of by Proposition 2.1, we have 


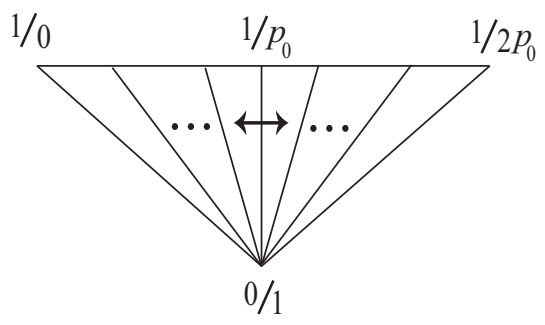

(i) $p=2 p_{0}$

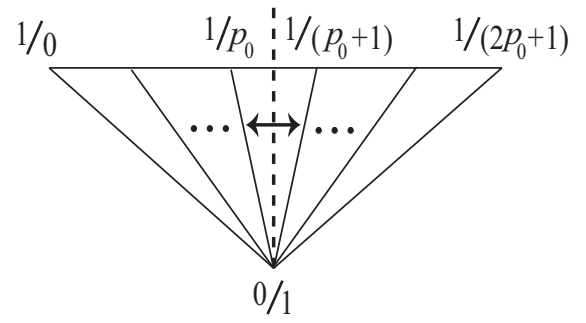

(ii) $p=2 p+1$

Figure 2. The involution $\tau$

only to show that $\tau$ preserves the homotopy classes, in $S^{3}-K(1 / p)$, of simple loops in $\boldsymbol{S}$. Since the reflection $\gamma_{0} \in \hat{\Gamma}_{1 / p}$ in the Farey edge $\langle\infty, 0\rangle$ has this property by Proposition 2.1, we may prove that $\psi:=\tau \circ \gamma_{0}$ has this property. Since $\psi$ is the parabolic transformation of $\mathcal{D}$, centered on 0 , by $p$ units in the counter-clockwise direction, it is induced by a self-homeomorphism $\tilde{\psi}$ of $\boldsymbol{S}$ which is the $p$ half twists along the simple loop $\alpha_{0}$. We may assume that the the half twists are made along a parallel copy of $\alpha_{0}$ lying in the interior of the lower hemisphere so that the support of $\tilde{\psi}$ is contained in the lower hemisphere. Then the inclusion map $j: \boldsymbol{S} \rightarrow S^{3}-K(1 / p)$ is homotoped to $j \circ \tilde{\psi}$ by pushing the lower-hemisphere sweeping out the inner tangle $\left(B^{3}, t(1 / p)\right)$, then through the upper hemisphere back to itself sweeping out the outer tangle $\left(B^{3}, t(\infty)\right)$, as in Figure 3(b). Hence we obtain the the desired result as in the proof of Proposition 2.1.

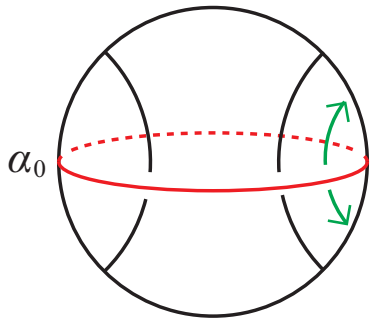

(a) $\left(B^{3}, t(\infty)\right)$

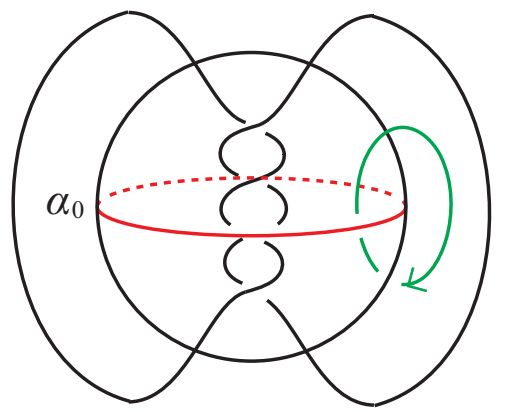

(b) $\left(S^{3}, K(1 / p)\right)$

FiguRE 3. 
In the following, we give a reformulation of the main theorem so that it is more suitable for the proof of the only if part. Suppose that $r$ is a rational number with $0<r<1$. Write

$$
r=\frac{1}{m_{1}+\frac{1}{m_{2}+\cdot \cdot+\frac{1}{m_{k}}}}=:\left[m_{1}, m_{2}, \ldots, m_{k}\right]
$$

where $k \geq 1,\left(m_{1}, \ldots, m_{k}\right) \in\left(\mathbb{Z}_{+}\right)^{k}$, and $m_{k} \geq 2$. Recall that the region, $R$, bounded by a pair of Farey edges with an endpoint $\infty$ and a pair of Farey edges with an endpoint $r$ forms a fundamental domain of the action of $\hat{\Gamma}_{r}$ on $\mathbb{H}^{2}$ (see Figure 1). Let $I_{1}(r)$ and $I_{2}(r)$ be the closed intervals in $\hat{\mathbb{R}}$ obtained as the intersection with $\hat{\mathbb{R}}-\{r, \infty\}$ of the closure of $R$. To be precise, $I_{1}(r)=\left[0, r_{1}\right]$ and $I_{2}(r)=\left[r_{2}, 1\right]$, where

$$
\left.\begin{array}{l}
r_{1}=\left[m_{1}, m_{2}, \ldots, m_{k-1}\right] \\
r_{2}=\left[m_{1}, m_{2}, \ldots, m_{k-1}, m_{k}-1\right] \\
r_{1}=\left[m_{1}, m_{2}, \ldots, m_{k-1}, m_{k}-1\right] \\
r_{2}=\left[m_{1}, m_{2}, \ldots, m_{k-1}\right]
\end{array}\right\} \quad \text { if } k \text { is odd }
$$

If $r=1 / p(p \geq 2)$, then $I_{1}(r)$ is degenerate to the singleton $\{0\}$. And if $r=(p-1) / p(p \geq 2)$, then $I_{2}(r)$ is degenerate to the singleton $\{1\}$. Otherwise, $I_{1}(r)$ and $I_{2}(r)$ are non-degenerate intervals, and the union $I_{1}(r) \cup I_{2}(r)$ forms a fundamental domain of the action of $\hat{\Gamma}_{r}$ on the domain of discontinuity of $\hat{\Gamma}_{r}$, the complement in $\hat{\mathbb{R}}$ of the closure of $\hat{\Gamma}_{r}\{\infty, r\}$. (In the exceptional case $r=1 / p$ (resp., $(p-1) / p$ ), the rational number 0 (resp., 1 ) lies in the limit set and $I_{2}(r)$ (resp., $I_{1}(r)$ ) is a fundamental domain of the action of $\hat{\Gamma}_{r}$ on the domain of discontinuity.)

Lemma 2.5. [6, Lemma 7.1] Suppose $0<r<1$. Then, for any $s \in \hat{\mathbb{Q}}$, there is a unique rational number $s_{0} \in I_{1}(r) \cup I_{2}(r) \cup\{\infty, r\}$ such that $s$ is contained in the $\hat{\Gamma}_{r}$-orbit of $s_{0}$, and in particular, $\alpha_{s}$ is homotopic to $\alpha_{s_{0}}$ in $S^{3}-K(r)$.

Thus the only if part of Theorem 2.3 when $r \notin \mathbb{Z} \cup\{\infty\}$ is equivalent to the following theorem.

Theorem 2.6. [6, Theorem 7.2] Suppose $0<r<1$. Then, for any rational number $s \in I_{1}(r) \cup I_{2}(r), \alpha_{s}$ is not null-homotopic in $S^{3}-K(r)$.

We can now reformulate our main theorem. 
Main Theorem 2.7. Let $p \geq 2$ be an integer. Then, for two distinct rational numbers $s, s^{\prime} \in I_{1}(1 / p) \cup I_{2}(1 / p)=\{0\} \cup\left[\frac{1}{p-1}, 1\right]$, the unoriented loops $\alpha_{s}$ and $\alpha_{s^{\prime}}$ are homotopic in $S^{3}-K(1 / p)$ if and only if $s^{\prime}=\tau(s)$, or in other words $s=q_{1} / p_{1}$ and $s^{\prime}=q_{2} / p_{2}$ satisfy $q_{1}=q_{2}$ and $q_{1} /\left(p_{1}+p_{2}\right)=1 / p$, where $\left(p_{i}, q_{i}\right)$ is a pair of relatively prime positive integers.

We prove the only if part by interpreting the situation in terms of combinatorial group theory. In other words, we prove that two words representing the free homotopy classes of $\alpha_{s}$ and $\alpha_{s^{\prime}}$ are conjugate in the 2-bridge link group $G(K(1 / p))$ only if $s$ and $s^{\prime}$ satisfy the conditions given in the statement of the theorem. The key tool used in the proof is small cancellation theory, which is one of the representative geometric techniques in combinatorial group theory, applied to reduced annular diagrams over 2-bridge link groups. The proof is contained in Section 5 .

\section{Preliminaries}

In this section, we introduce the upper presentation of a 2-bridge link group, and recall key facts established in [6] concerning it. These facts are to be used throughout this series of papers.

To find a presentation of the 2-bridge link group $G(K(r))$ explicitly, let $a$ and $b$, respectively, be the elements of $\pi_{1}\left(B^{3}-t(\infty), x_{0}\right)$ represented by the oriented loops $\mu_{1}$ and $\mu_{2}$ based on $x_{0}$ as illustrated in Figure 4. Then $\{a, b\}$ forms the meridian pair of $\pi_{1}\left(B^{3}-t(\infty)\right)$, which is identified with the free group $F(a, b)$. Note that $\mu_{i}$ intersects the disk, $\delta_{i}$, in $B^{3}$ bounded by a component of $t(\infty)$ and the essential arc, $\gamma_{i}$, on $\partial\left(B^{3}, t(\infty)\right)=\left(\boldsymbol{S}^{2}, \boldsymbol{P}\right)$ of slope $1 / 0$, in Figure 4. Obtain a word $u_{r}$ in $\{a, b\}$ by reading the intersection of the (suitably oriented) loop $\alpha_{r}$ with $\gamma_{1} \cup \gamma_{2}$, where a positive intersection with $\gamma_{1}$ (resp., $\gamma_{2}$ ) corresponds to $a$ (resp., $b$ ). Then the cyclic word $\left(u_{r}\right)$ represents the free homotopy class of the (oriented) loop $\alpha_{r}$ (see the paragraph preceding Definition 3.3 for the precise definition of a cyclic word). It then follows that

$$
\begin{aligned}
G(K(r)) & =\pi_{1}\left(S^{3}-K(r)\right) \cong \pi_{1}\left(B^{3}-t(\infty)\right) /\left\langle\left\langle\alpha_{r}\right\rangle\right\rangle \\
& \cong F(a, b) /\left\langle\left\langle u_{r}\right\rangle\right\rangle \cong\left\langle a, b \mid u_{r}\right\rangle .
\end{aligned}
$$

This one-relator presentation is called the upper presentation of $G(K(r))$ (see [2]). If $r \neq \infty$, then $\alpha_{r}$ intersects $\gamma_{1}$ and $\gamma_{2}$ alternately, and hence $a$ and $b$ appear in $\left(u_{r}\right)$ alternately (with exponents \pm 1 ). It is known by [14, Proposition 1] that there is a nice formula to find $u_{r}$ as follows (see [6, Remark 1] for a geometric picture). 


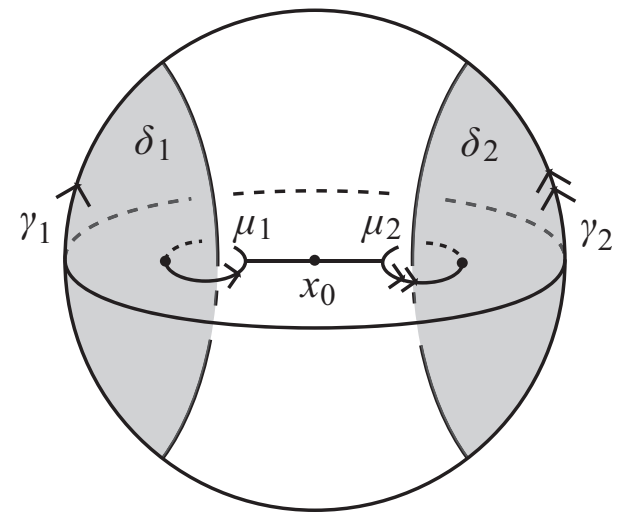

Figure 4. $\pi_{1}\left(B^{3}-t(\infty), x_{0}\right)=F(a, b)$, where $a$ and $b$ are represented by $\mu_{1}$ and $\mu_{2}$, respectively.

Lemma 3.1. Let $p$ and $q$ be relatively prime positive integers such that $p \geq 1$. For $1 \leq i \leq p-1$, let

$$
\epsilon_{i}=(-1)^{\lfloor i q / p\rfloor}
$$

where $\lfloor x\rfloor$ is the greatest integer not exceeding $x$.

(1) If $p$ is odd, then

$$
u_{q / p}=a \hat{u}_{q / p} b^{(-1)^{q}} \hat{u}_{q / p}^{-1},
$$

where $\hat{u}_{q / p}=b^{\epsilon_{1}} a^{\epsilon_{2}} \cdots b^{\epsilon_{p-2}} a^{\epsilon_{p-1}}$.

(2) If $p$ is even, then

$$
u_{q / p}=a \hat{u}_{q / p} a^{-1} \hat{u}_{q / p}^{-1},
$$

where $\hat{u}_{q / p}=b^{\epsilon_{1}} a^{\epsilon_{2}} \cdots a^{\epsilon_{p-2}} b^{\epsilon_{p-1}}$

Remark 3.2. For $r=0 / 1$ and $r=1 / 0$, we have $u_{0 / 1}=a b$ and $u_{1 / 0}=1$.

3.1. $S$ - and $T$-sequences of slope $r$. We define the sequence $S(r)$ and the cyclic sequence $C S(r)$ of slope $r$ both of which arise from the single relator $u_{r}$ of the upper presentation $G(K(r))=\left\langle a, b \mid u_{r}\right\rangle$, and review several important properties of these sequences from [6] so that we can adapt small cancellation theory in Section 4 .

We first fix some definitions and notation. Let $X$ be a set. By a word in $X$, we mean a finite sequence $x_{1}^{\epsilon_{1}} x_{2}^{\epsilon_{2}} \cdots x_{n}^{\epsilon_{n}}$ where $x_{i} \in X$ and $\epsilon_{i}= \pm 1$. Here we call $x_{i}^{\epsilon_{i}}$ the $i$-th letter of the word. For two words $u, v$ in $X$, by $u \equiv v$ we denote the visual equality of $u$ and $v$, meaning that if $u=x_{1}^{\epsilon_{1}} \cdots x_{n}^{\epsilon_{n}}$ and $v=y_{1}^{\delta_{1}} \cdots y_{m}^{\delta_{m}}\left(x_{i}, y_{j} \in X ; \epsilon_{i}, \delta_{j}= \pm 1\right)$, then $n=m$ and $x_{i}=y_{i}$ and $\epsilon_{i}=\delta_{i}$ for each $i=1, \ldots, n$. For example, two words $x_{1} x_{2} x_{2}^{-1} x_{3}$ and $x_{1} x_{3}\left(x_{i} \in X\right)$ 
are not visually equal, though $x_{1} x_{2} x_{2}^{-1} x_{3}$ and $x_{1} x_{3}$ are equal as elements of the free group with basis $X$. The length of a word $v$ is denoted by $|v|$. A word $v$ in $X$ is said to be reduced if $v$ does not contain $x x^{-1}$ or $x^{-1} x$ for any $x \in X$. A word is said to be cyclically reduced if all its cyclic permutations are reduced. A cyclic word is defined to be the set of all cyclic permutations of a word. By $(v)$ we denote the cyclic word associated with a word $v$. Also by $(u) \equiv(v)$ we mean the visual equality of two cyclic words $(u)$ and $(v)$. In fact, $(u) \equiv(v)$ if and only if $v$ is visually a cyclic shift of $u$.

Definition 3.3. (1) Let $v$ be a reduced word in $\{a, b\}$. Decompose $v$ into

$$
v \equiv v_{1} v_{2} \cdots v_{t}
$$

where, for each $i=1, \ldots, t-1$, all letters in $v_{i}$ have positive (resp., negative) exponents, and all letters in $v_{i+1}$ have negative (resp., positive) exponents. Then the sequence of positive integers $S(v):=\left(\left|v_{1}\right|,\left|v_{2}\right|, \ldots,\left|v_{t}\right|\right)$ is called the $S$-sequence of $v$.

(2) Let $(v)$ be a reduced cyclic word in $\{a, b\}$. Decompose $(v)$ into

$$
(v) \equiv\left(v_{1} v_{2} \cdots v_{t}\right),
$$

where all letters in $v_{i}$ have positive (resp., negative) exponents, and all letters in $v_{i+1}$ have negative (resp., positive) exponents (taking subindices modulo $t$ ). Then the cyclic sequence of positive integers $C S(v):=\left(\left(\left|v_{1}\right|,\left|v_{2}\right|, \ldots,\left|v_{t}\right|\right)\right)$ is called the cyclic $S$-sequence of $(v)$. Here the double parentheses denote that the sequence is considered modulo cyclic permutations.

(3) A reduced word $v$ in $\{a, b\}$ is said to be alternating if $a^{ \pm 1}$ and $b^{ \pm 1}$ appear in $v$ alternately, i.e., neither $a^{ \pm 2}$ nor $b^{ \pm 2}$ appears in $v$. A cyclic word $(v)$ is said to be alternating if all cyclic permutations of $v$ are alternating. In the latter case, we also say that $v$ is cyclically alternating.

Definition 3.4. For a rational number $r$ with $0<r \leq 1$, let $G(K(r))=$ $\left\langle a, b \mid u_{r}\right\rangle$ be the upper presentation. Then the symbol $S(r)$ (resp., $C S(r)$ ) denotes the $S$-sequence $S\left(u_{r}\right)$ of $u_{r}$ (resp., cyclic $S$-sequence $C S\left(u_{r}\right)$ of $\left(u_{r}\right)$ ), which is called the $S$-sequence of slope $r$ (resp., the cyclic $S$-sequence of slope $r)$.

Lemma 3.5. 6, Proposition 4.1 and Lemma 5.2] (1) An alternating word in $\{a, b\}$ is completely determined by the initial letter and the associated $S$ sequence.

(2) Suppose that $r$ is a rational number with $0<r \leq 1$. Let $w$ be an arbitrary cyclic permutation of the single relator $u_{r}$ of the upper presentation 
of $G(K(r))$. Then the set

\{the initial letter of $w^{\prime} \mid\left(w^{\prime}\right) \equiv\left(u_{r}^{ \pm 1}\right)$ and $\left.S\left(w^{\prime}\right)=S(w)\right\}$

equals $\left\{a, a^{-1}, b, b^{-1}\right\}$.

The above lemma implies that the cyclic word $\left(u_{r}^{ \pm 1}\right)$ is completely determined by the cyclic $S$-sequence $C S(r)$, to be precise, an alternating word $w^{\prime}$ is a cyclic permutation of $u_{r}^{ \pm 1}$ if and only if $w^{\prime}$ satisfies $S\left(w^{\prime}\right)=S(w)$ for some $w$ a cyclic permutation of $u_{r}$.

Lemma 3.6. [6, Lemma 4.8 and Proposition 4.2] Suppose that $r=q / p$ is a rational number with $0<r \leq 1$, where $p$ and $q$ are relatively prime positive integers. Then the following hold.

(1) The sequence $S(r)$ has length $2 q$, and its $j$-th term $\nu_{j}(r)$ is given by the following formula $(1 \leq j \leq 2 q)$ :

$$
\nu_{j}(r)=\lfloor j p / q\rfloor_{*}-\lfloor(j-1) p / q\rfloor_{*},
$$

where $\lfloor x\rfloor_{*}$ is the greatest integer (strictly) smaller than $x$.

(2) The sequence $S(r)$ represents the cyclic sequence $C S(r)$. Moreover the cyclic sequence $C S(r)$ is invariant by the half-rotation; that is, if $\nu_{j}(r)$ denotes the $j$-th term of $S(r)(1 \leq j \leq 2 q)$, then $\nu_{j}(r)=\nu_{q+j}(r)$ for every integer $j(1 \leq j \leq q)$.

Remark 3.7. For $r=0$, we have $S\left(u_{0}\right)=(2)$ by Remark 3.2.

In the remainder of this paper unless specified otherwise, we suppose that $r$ is a rational number with $0<r \leq 1$, and write $r$ as a continued fraction:

$$
r=\left[m_{1}, m_{2}, \ldots, m_{k}\right],
$$

where $k \geq 1,\left(m_{1}, \ldots, m_{k}\right) \in\left(\mathbb{Z}_{+}\right)^{k}$ and $m_{k} \geq 2$ unless $k=1$. For brevity, we write $m$ for $m_{1}$.

Lemma 3.8. [6, Proposition 4.3] The following hold.

(1) Suppose $k=1$, i.e., $r=1 / m$. Then $S(r)=(m, m)$.

(2) Suppose $k \geq 2$. Then each term of $S(r)$ is either $m$ or $m+1$, and $S(r)$ begins with $m+1$ and ends with $m$. Moreover, the following hold.

(a) If $m_{2}=1$, then no two consecutive terms of $S(r)$ can be $(m, m)$, so there is a sequence of positive integers $\left(t_{1}, t_{2}, \ldots, t_{s}\right)$ such that

$$
S(r)=\left(t_{1}\langle m+1\rangle, m, t_{2}\langle m+1\rangle, m, \ldots, t_{s}\langle m+1\rangle, m\right) .
$$

Here, the symbol ' $t_{i}\langle m+1\rangle$ " represents $t_{i}$ successive $m+1$ 's. 
(b) If $m_{2} \geq 2$, then no two consecutive terms of $S(r)$ can be $(m+1, m+$ $1)$, so there is a sequence of positive integers $\left(t_{1}, t_{2}, \ldots, t_{s}\right)$ such that

$$
S(r)=\left(m+1, t_{1}\langle m\rangle, m+1, t_{2}\langle m\rangle, \ldots, m+1, t_{s}\langle m\rangle\right) .
$$

Here, the symbol ' $t_{i}\langle m\rangle$ " represents $t_{i}$ successive $m$ 's.

Definition 3.9. If $k \geq 2$, the symbol $T(r)$ denotes the sequence $\left(t_{1}, t_{2}, \ldots, t_{s}\right)$ in Lemma 3.8, which is called the T-sequence of slope $r$. The symbol $C T(r)$ denotes the cyclic sequence represented by $T(r)$, which is called the cyclic T-sequence of slope $r$.

Example 3.10. (1) Let $r=10 / 37=[3,1,2,3]$. By Lemma 3.1, we see that the $S$-sequence of $\hat{u}_{r}$ is

$$
S\left(\hat{u}_{r}\right)=(3,4,4,3,4,4,3,4,4,3) .
$$

By the formula for $u_{r}$ in Lemma 3.1, this implies

$$
S(r)=S\left(u_{r}\right)=(\underbrace{4,4,4}_{3}, 3, \underbrace{4,4}_{2}, 3, \underbrace{4,4}_{2}, 3, \underbrace{4,4,4}_{3}, 3, \underbrace{4,4}_{2}, 3, \underbrace{4,4}_{2}, 3) .
$$

So $T(r)=(3,2,2,3,2,2)$ and $C T(r)=((3,2,2,3,2,2))$.

(2) Let $r=8 / 35=[4,2,1,2]$. Again by Lemma 3.1, we obtain that the $S$-sequence of $\hat{u}_{r}$ is

$$
S\left(\hat{u}_{r}\right)=(4,4,5,4,4,5,4,4) .
$$

By the formula for $u_{r}$ in Lemma 3.1, this implies

$$
S(r)=S\left(u_{r}\right)=(5, \underbrace{4}_{1}, 5, \underbrace{4,4}_{2}, 5, \underbrace{4,4}_{2}, 5, \underbrace{4}_{1}, 5, \underbrace{4,4}_{2}, 5, \underbrace{4,4}_{2}) .
$$

So $T(r)=(1,2,2,1,2,2)$ and $C T(r)=((1,2,2,1,2,2))$.

Lemma 3.11. [6, Proposition 4.4] Let $\tilde{r}$ be the rational number defined as

$$
\tilde{r}= \begin{cases}{\left[m_{3}, \ldots, m_{k}\right]} & \text { if } m_{2}=1 \\ {\left[m_{2}-1, m_{3}, \ldots, m_{k}\right]} & \text { if } m_{2} \geq 2\end{cases}
$$

Then we have

$$
T(r)= \begin{cases}S(\tilde{r}) & \text { if } m_{2}=1 \\ \overleftarrow{S}(\tilde{r}) & \text { if } m_{2} \geq 2\end{cases}
$$

where $\overleftarrow{S}(\tilde{r})$ denotes the sequence obtained from $S(\tilde{r})$ reversing its order

Proposition 3.12. [6, Proposition 4.5] The sequence $S(r)$ has a unique decomposition $\left(S_{1}, S_{2}, S_{1}, S_{2}\right)$ which satisfies the following. 
(1) Each $S_{i}$ is symmetric, i.e., the sequence obtained from $S_{i}$ by reversing the order is equal to $S_{i}$. (Here, $S_{1}$ is empty if $k=1$.)

(2) Each $S_{i}$ (if it is not empty) occurs only twice in the cyclic sequence $C S(r)$.

(3) $S_{1}$ (if it is not empty) begins and ends with $m+1$.

(4) $S_{2}$ begins and ends with $m$.

Example 3.13. (1) Let $r=10 / 37=[3,1,2,3]$. Recall from Example 3.10 that

$$
S(r)=(4,4,4,3,4,4,3,4,4,3,4,4,4,3,4,4,3,4,4,3) .
$$

Putting $S_{1}=(4,4,4)$ and $S_{2}=(3,4,4,3,4,4,3)$, we have

$$
S(r)=\left(S_{1}, S_{2}, S_{1}, S_{2}\right),
$$

where $S_{1}$ and $S_{2}$ satisfy all the assertions in Proposition 3.12,

(2) Let $r=8 / 35=[4,2,1,2]$. Recall also from Example 3.10 that

$$
S(r)=(5,4,5,4,4,5,4,4,5,4,5,4,4,5,4,4) .
$$

Putting $S_{1}=(5,4,5)$ and $S_{2}=(4,4,5,4,4)$, we also have

$$
S(r)=\left(S_{1}, S_{2}, S_{1}, S_{2}\right),
$$

where $S_{1}$ and $S_{2}$ satisfy all the assertions in Proposition 3.12 .

Corollary 3.14. [6, Corollary 4.6] The cyclic S-sequence CS $(r)$ is symmetric, i.e., the cyclic sequence obtained from $C S(r)$ by reversing its cyclic order is equivalent to $C S(r)$ (as a cyclic sequence). In particular, in Lemma 3.11, we actually have

$$
C T(r)=C S(\tilde{r}) .
$$

Remark 3.15. By using the fact that $u_{r}$ is obtained from the line of slope $r$ in $\mathbb{R}^{2}-\mathbb{Z}^{2}$ by reading its intersection with the vertical lattice lines, we see that the slope $s=q / p$ is recovered from $C S(s)=\left(\left(S_{1}, S_{2}, S_{1}, S_{2}\right)\right)$ by the rule that $p$ is the sum of the terms of $S_{1}$ and $S_{2}$ whereas $q$ is the sum of the lengths of $S_{1}$ and $S_{2}$.

Lemma 3.16. [6, Proof of Proposition 4.5] Let $\tilde{r}$ be the rational number defined as in Lemma 3.11. Also let $S(\tilde{r})=\left(T_{1}, T_{2}, T_{1}, T_{2}\right)$ and $S(r)=\left(S_{1}, S_{2}, S_{1}, S_{2}\right)$ be decompositions described as in Proposition 3.12. Then the following hold.

(1) If $m_{2}=1$ and $k=3$, then $T_{1}=\emptyset, T_{2}=\left(m_{3}\right)$, and $S_{1}=\left(m_{3}\langle m+1\rangle\right)$, $S_{2}=(m)$. 
(2) If $m_{2}=1$ and $k \geq 4$, then $T_{1}=\left(t_{1}, \ldots, t_{s_{1}}\right), T_{2}=\left(t_{s_{1}+1}, \ldots, t_{s_{2}}\right)$, and

$$
S_{1}=\left(t_{1}\langle m+1\rangle, m, t_{2}\langle m+1\rangle, \ldots, t_{s_{1}-1}\langle m+1\rangle, m, t_{s_{1}}\langle m+1\rangle\right),
$$$$
S_{2}=\left(m, t_{s_{1}+1}\langle m+1\rangle, m, \ldots, m, t_{s_{2}}\langle m+1\rangle, m\right) \text {. }
$$

(3) If $k=2$, then $T_{1}=\emptyset, T_{2}=\left(m_{2}-1\right)$, and $S_{1}=(m+1), S_{2}=$ $\left(\left(m_{2}-1\right)\langle m\rangle\right)$.

(4) If $m_{2} \geq 2$ and $k \geq 3$, then $T_{1}=\left(t_{1}, \ldots, t_{s_{1}}\right), T_{2}=\left(t_{s_{1}+1}, \ldots, t_{s_{2}}\right)$, and

$$
\begin{aligned}
& S_{1}=\left(m+1, t_{s_{1}+1}\langle m\rangle, m+1, \ldots, m+1, t_{s_{2}}\langle m\rangle, m+1\right), \\
& S_{2}=\left(t_{1}\langle m\rangle, m+1, t_{2}\langle m\rangle, \ldots, t_{s_{1}-1}\langle m\rangle, m+1, t_{s_{1}}\langle m\rangle\right) .
\end{aligned}
$$

The following example will be useful in the second [7] of this series of papers.

Example 3.17. (1) If $r=[2,1, n]$ with $n \geq 2$, then $\tilde{r}=[n]$ by Lemma 3.11. So by Lemma $3.8(1), S(\tilde{r})=(\emptyset, n, \emptyset, n)$. Thus by Lemma $3.16(1), S(r)=$ $(n\langle 3\rangle, 2, n\langle 3\rangle, 2)$, where $S_{1}=(n\langle 3\rangle)$ and $S_{2}=(2)$.

(2) If $r=[2, n]$ with $n \geq 2$, then $\tilde{r}=[n-1]$ by Lemma 3.11. So by Lemma 3.8 (1), $S(\tilde{r})=(\emptyset, n-1, \emptyset, n-1)$. Thus by Lemma 3.16(3), $S(r)=$ $(3,(n-1)\langle 2\rangle, 3,(n-1)\langle 2\rangle)$, where $S_{1}=(3)$ and $S_{2}=((n-1)\langle 2\rangle)$.

By Lemmas 3.8 and 3.16, we can easily observe the following lemma.

Lemma 3.18. Let $S(r)=\left(S_{1}, S_{2}, S_{1}, S_{2}\right)$ be as in Proposition 3.12. Then the following hold.

(1) If $m_{2}=1$, then $(m+1, m+1)$ appears in $S_{1}$.

(2) If $m_{2} \geq 2$ and if $r \neq[m, 2]=2 /(2 m+1)$, then $(m, m)$ appears in $S_{2}$.

The following is a refinement of [6, Lemma 7.3 and Remark 5].

Proposition 3.19. Suppose that $r$ is a rational number with $0<r<1$. Let $S(r)=\left(S_{1}, S_{2}, S_{1}, S_{2}\right)$ be as in Proposition 3.12. For a rational number s with $0<s \leq 1$, the following hold.

(1) If $C S(s)$ contains both $S_{1}$ and $S_{2}$ as subsequences, then $s \notin I_{1}(r) \cup I_{2}(r)$.

(2) If $r=1 / p(p \geq 2)$ and $s \in I_{1}(r) \cup I_{2}(r)$, then $C S(s)$ consists of integers less than $p$.

In the above proposition (and throughout this series of papers), we mean by a subsequence a subsequence without leap. Namely a sequence $\left(a_{1}, a_{2}, \ldots, a_{l}\right)$ is called a subsequence of a cyclic sequence, if there is a sequence $\left(b_{1}, b_{2}, \ldots, b_{n}\right)$ representing the cyclic sequence such that $l \leq n$ and $a_{i}=b_{i}$ for $1 \leq i \leq l$.

Proof. (1) Suppose that $C S(s)$ contains both $S_{1}$ and $S_{2}$ as subsequences. Recall that $r=\left[m_{1}, m_{2}, \ldots, m_{k}\right]$. Write $s=\left[n_{1}, n_{2}, \ldots, n_{t}\right]$, where $t \geq 1$, 
$\left(n_{1}, \ldots, n_{t}\right) \in\left(\mathbb{Z}_{+}\right)^{t}$ and $n_{t} \geq 2$ unless $t=1$. We show that the following three conditions hold by induction on $k \geq 1$, refining the proof of [6, Lemma 7.3]. (As noted in [6, Remark 5], this is equivalent to the desired conclusion that $s \notin I_{1}(r) \cup I_{2}(r)$.)

(i) $t \geq k$.

(ii) $n_{i}=m_{i}$ for each $i=1, \ldots, k-1$.

(iii) Either $n_{k} \geq m_{k}$ or both $n_{k}=m_{k}-1$ and $t>k$.

First let $k=1$. Then $r=[m]$ and $S(r)=(m, m)=\left(S_{2}, S_{2}\right)$, where $S_{1}$ is empty. By hypothesis, $C S(s)$ contains a term $m$. So if $t=1$, then $n_{1}=m$, while if $t \geq 2$, then $n_{1}$ is either $m$ or $m-1$. Thus the three conditions hold, proving the base step.

Now let $k \geq 2$. Then $C S(r)=\left(\left(S_{1}, S_{2}, S_{1}, S_{2}\right)\right)$ consists of $m$ and $m+1$ by Lemma 3.8. This yields that $C S(s)$ consists of $m$ and $m+1$. This happens only when $t \geq 2$ and $n_{1}=m_{1}$. For the rational numbers $r$ and $s$, define the rational numbers $\tilde{r}$ and $\tilde{s}$ as in Lemma 3.11 .

We consider three cases separately.

Case 1. $m_{2}=1$.

In this case, $k \geq 3$ and, by Lemma $3.18,(m+1, m+1)$ appears in $S_{1}$ as a subsequence, so in $C S(s)$ as a subsequence. Thus by Lemma 3.8, $n_{2}=1$ and so $t \geq 3$. So, we have

$$
\tilde{r}=\left[m_{3}, \ldots, m_{k}\right] \text { and } \tilde{s}=\left[n_{3}, \ldots, n_{t}\right] .
$$

Let $S(\tilde{r})=\left(T_{1}, T_{2}, T_{1}, T_{2}\right)$ be the decomposition of $S(\tilde{r})$ given by Proposition 3.12 .

Case 1.a. $k=3$.

By Lemma $3.16(1), S_{1}=\left(m_{3}\langle m+1\rangle\right)$ and $S_{2}=(m)$. Since $S_{1}$ is contained in $C S(s)$ by assumption, $C S(\tilde{s})=C T(s)$ contains a term $m_{3}+d$ for some $d \in \mathbb{Z}_{+} \cup\{0\}$. If $t=3$ then $C S(\tilde{s})=\left(\left(n_{3}, n_{3}\right)\right)$ and hence we have $n_{3} \geq m_{3}$. If $t \geq 4$ then $C S(\tilde{s})$ consists of $n_{3}$ and $n_{3}+1$ and hence $n_{3} \geq m_{3}-1$. Thus in either case, the three conditions hold, as desired.

Case 1.b. $k \geq 4$.

Since $S_{2}$ is contained in $C S(s)$ by assumption and since $S_{2}$ begins and ends with $m$, we see by Lemma 3.16) (2) that $C S(\tilde{s})=C T(s)$ contains $T_{2}$. Similarly, by using the assumption that $S_{1}$ is contained in $C S(s)$, we see that $C S(\tilde{s})=C T(s)$ contains a subsequence of the form

$$
\left(t_{1}+d^{\prime}, t_{2}, \ldots, t_{s_{1}-1}, t_{s_{1}}+d^{\prime \prime}\right),
$$


where $\left(t_{1}, t_{2}, \ldots, t_{s_{1}-1}, t_{s_{1}}\right)=T_{1}$ and $d^{\prime}, d^{\prime \prime} \in \mathbb{Z}_{+} \cup\{0\}$. Since $t_{1}=t_{s_{1}}=$ $m_{3}+1$ by Proposition 3.12 , this actually implies that $C S(\tilde{s})$ contains $T_{1}$ as a subsequence. Thus $C S(\tilde{s})$ contains both $T_{1}$ and $T_{2}$ as subsequences. Since $S(\tilde{r})=\left(T_{1}, T_{2}, T_{1}, T_{2}\right)$ is the decomposition described as in Proposition 3.12 , the inductive hypothesis implies that the following three conditions hold.

(i) $t \geq k$.

(ii) $n_{i}=m_{i}$ for each $i=3, \ldots, k-1$.

(iii) Either $n_{k} \geq m_{k}$ or both $n_{k}=m_{k}-1$ and $t>k$.

Since $n_{1}=m_{1}$ and $n_{2}=m_{2}$, this implies that the original three conditions hold, as desired.

Case 2. Both $m_{2}=2$ and $k=2$.

In this case, the three conditions always hold, because if $n_{2}=1$ then we must have $t \geq 3$, otherwise $n_{2} \geq 2=m_{2}$. (Recall that we already proved that $n_{1}=m_{1}$.)

Case 3. Either $m_{2} \geq 3$ or both $m_{2}=2$ and $k \geq 3$.

In this case, by Lemma $3.18,(m, m)$ appears in $S_{2}$ as a subsequence, so in $C S(s)$ as a subsequence. Thus $n_{2} \geq 2$ by Lemma 3.8, and so we have

$$
\tilde{r}=\left[m_{2}-1, m_{3}, \ldots, m_{k}\right] \quad \text { and } \quad \tilde{s}=\left[n_{2}-1, n_{3}, \ldots, n_{t}\right] .
$$

Let $S(\tilde{r})=\left(T_{1}, T_{2}, T_{1}, T_{2}\right)$ be the decomposition of $S(\tilde{r})$ given by Proposition 3.12, Since $S_{1}$ is contained in $C S(s)$ by assumption and since $S_{1}$ begins and ends with $m+1$, we see by Lemma 3.16) (4) that $C S(\tilde{s})=C T(s)$ contains $T_{2}$. Similarly, by using the assumption that $S_{2}$ is contained in $C S(s)$, we see that $C S(\tilde{s})=C T(s)$ contains a subsequence of the form

$$
\left(t_{1}+d^{\prime}, t_{2}, \ldots, t_{s_{1}-1}, t_{s_{1}}+d^{\prime \prime}\right),
$$

where $\left(t_{1}, t_{2}, \ldots, t_{s_{1}-1}, t_{s_{1}}\right)=T_{1}$ and $d^{\prime}, d^{\prime \prime} \in \mathbb{Z}_{+} \cup\{0\}$. Since $t_{1}=t_{s_{1}}=\left(m_{2}-\right.$ $1)+1=m_{2}$ by Proposition 3.12 , this actually implies that $C S(\tilde{s})$ contains $T_{1}$ as a subsequence. Thus $C S(\tilde{s})$ contains both $T_{1}$ and $T_{2}$ as subsequences. Since $S(\tilde{r})=\left(T_{1}, T_{2}, T_{1}, T_{2}\right)$ is the decomposition described as in Proposition 3.12 , the inductive hypothesis implies that the following three conditions hold.

(i) $t \geq k$.

(ii) $n_{i}=m_{i}$ for each $i=2, \ldots, k-1$.

(iii) Either $n_{k} \geq m_{k}$ or both $n_{k}=m_{k}-1$ and $t>k$.

Since $n_{1}=m_{1}$, this implies that the original three conditions hold, as desired.

(2) Assume $r=1 / p$. Since $0 \neq s \in I_{1}(1 / p) \cup I_{2}(1 / p)=\{0\} \cup\left[\frac{1}{p-1}, 1\right], s$ has a continued fraction expansion $s=\left[n_{1}, \ldots, n_{t}\right]$ such that either both $n_{1}=p-1$ 
and $t=1$ or both $0<n_{1} \leq p-2$ and $t \geq 1$. If $n_{1}=p-1$ and $t=1$, then $s=1 /(p-1)$ and $C S(s)=((p-1, p-1))$; so the assertion holds. If $0<n_{1} \leq p-2$ and $t \geq 1$, then each term of $C S(s)$ is equal to $n_{1} \leq p-2$ or $n_{1}+1 \leq p-1$ by Lemma 3.8, so the assertion holds.

Remark 3.20. If $r \neq 1 / 2$, then the conclusions of Proposition 3.19 hold even for $s=0$. In fact, if $r \neq 1 / 2$, then either (i) $r=1 / p$ with $p \geq 3$ and so $S(r)=(p, p)=\left(S_{2}, S_{2}\right)$, where $S_{1}$ is empty, or (ii) both $S_{1}$ and $S_{2}$ are nonempty. Since $C S\left(u_{0}\right)=((2))$ by Remark 3.7, this implies the conclusions of Proposition 3.19 for $s=0$. However, if $r=1 / 2$, then none of the assertions of Proposition 3.19 holds for $s=0$.

3.2. Small cancellation conditions. We now recall the small cancellation conditions for 2-bridge link groups established in [6].

Let $F(X)$ be the free group with basis $X$. A subset $R$ of $F(X)$ is said to be symmetrized, if all elements of $R$ are cyclically reduced and, for each $w \in R$, all cyclic permutations of $w$ and $w^{-1}$ also belong to $R$.

Definition 3.21. Suppose that $R$ is a symmetrized subset of $F(X)$. A nonempty word $b$ is called a piece if there exist distinct $w_{1}, w_{2} \in R$ such that $w_{1} \equiv b c_{1}$ and $w_{2} \equiv b c_{2}$. The small cancellation conditions $C(i)$ and $T(j)$ on $R$, where $i$ and $j$ are integers such that $i \geq 2$ and $j \geq 3$, are defined as follows (see [10]).

(1) Condition $C(i)$ : If $w \in R$ is a product of $n$ pieces, then $n \geq i$.

(2) Condition $T(j)$ : For $w_{1}, \ldots, w_{n} \in R$ with no successive elements $w_{t}, w_{t+1}$ an inverse pair $(t \bmod n)$, if $n<j$, then at least one of the products $w_{1} w_{2}, \ldots, w_{n-1} w_{n}, w_{n} w_{1}$ is freely reduced without cancellation.

The following proposition enables us to apply the small cancellation theory to our problem.

Proposition 3.22. [6, Theorem 5.1] Suppose that $r$ is a rational number with $0<r<1$. Let $R$ be the symmetrized subset of $F(a, b)$ generated by the single relator $u_{r}$ of the upper presentation of $G(K(r))$. Then $R$ satisfies $C(4)$ and $T(4)$.

To conclude this section, we recall a key fact concerning the cyclic word $\left(u_{r}\right)$, which is used in the proof of the main theorem in Section 5. (In fact, it is also used in the proof of Proposition 3.22 above and that of Lemma 4.10 implicitly.)

Definition 3.23. For a positive integer $n$, a non-empty subword $w$ of the cyclic word $\left(u_{r}\right)$ is called a maximal $n$-piece if $w$ is a product of $n$ pieces and 
if any subword $w^{\prime}$ of $\left(u_{r}\right)$ which properly contains $w$ as an initial subword is not a product of $n$ pieces.

Lemma 3.24. [6, Corollary 5.4] Suppose that $r$ is a rational number such that $0<r<1$. Let $u_{r}$ be the single relator of the upper presentation of $G(K(r))$, and let $S(r)=\left(S_{1}, S_{2}, S_{1}, S_{2}\right)$ be as in Proposition 3.12. Decompose

$$
u_{r} \equiv v_{1} v_{2} v_{3} v_{4}
$$

where $S\left(v_{1}\right)=S\left(v_{3}\right)=S_{1}$ and $S\left(v_{2}\right)=S\left(v_{4}\right)=S_{2}$. Let $v_{i b}^{*}$ be the maximal proper initial subword of $v_{i}$, i.e., the initial subword of $v_{i}$ such that $\left|v_{i b}^{*}\right|=$ $\left|v_{i}\right|-1(i=1,2,3,4)$. Then the following hold, where $v_{i b}$ and $v_{i e}$ are nonempty initial and terminal subwords of $v_{i}$ with $\left|v_{i b}\right|,\left|v_{i e}\right| \leq\left|v_{i}\right|-1$, respectively.

(1) If $r=1 / p$ for some integer $p \geq 2$, then $v_{1}$ and $v_{3}$ are the empty words and the following hold.

(a) The following is the list of all maximal 1-pieces of $\left(u_{r}\right)$, arranged in the order of the position of the initial letter:

$$
v_{2 b}^{*}, v_{2 e}, v_{4 b}^{*}, v_{4 e} .
$$

(b) The following is the list of all maximal 2-pieces of $\left(u_{r}\right)$, arranged in the order of the position of the initial letter:

$$
v_{2}, v_{2 e} v_{4 b}^{*}, v_{4}, v_{4 e} v_{2 b}^{*} \text {. }
$$

(2) If $r \neq 1 / p$ for any integer $p \geq 2$, then the following hold.

(a) The following is the list of all maximal 1-pieces of $\left(u_{r}\right)$, arranged in the order of the position of the initial letter:

$$
v_{1 b}^{*}, v_{1 e} v_{2}, v_{2} v_{3 b}^{*}, v_{2 e} v_{3 b}^{*}, v_{3 b}^{*}, v_{3 e} v_{4}, v_{4} v_{1 b}^{*}, v_{4 e} v_{1 b}^{*} .
$$

(b) The following is the list of all maximal 2-pieces of $\left(u_{r}\right)$, arranged in the order of the position of the initial letter:

$v_{1} v_{2}, v_{1 e} v_{2} v_{3 b}^{*}, v_{2} v_{3} v_{4}, v_{2 e} v_{3} v_{4}, v_{3} v_{4}, v_{3 e} v_{4} v_{1 b}^{*}, v_{4} v_{1} v_{2}, v_{4 e} v_{1} v_{2}$.

The following corollary will be used in the sequels [7] and [8] of the present paper.

Corollary 3.25. If $r$ is a rational number such that $0<r<1$ and $r \neq 1 / p$ for any integer $p \geq 2$, then the following hold.

(1) A subword $w$ of the cyclic word $\left(u_{r}^{ \pm 1}\right)$ is a piece if and only if $S(w)$ does not contain $S_{1}$ as a subsequence and does not contain $S_{2}$ in its interior, i.e., $S(w)$ does not contain a subsequence $\left(\ell_{1}, S_{2}, \ell_{2}\right)$ for any $\ell_{1}, \ell_{2} \in \mathbb{Z}_{+}$. 
(2) For a subword $w$ of the cyclic word $\left(u_{r}^{ \pm 1}\right)$, if $S(w)$ either contains $\left(S_{1}, S_{2}\right)$ as a proper initial subsequence or contains $\left(S_{2}, S_{1}\right)$ as a proper terminal subsequence, then $w$ is not a product of two pieces.

Proof. (1) We prove the assertion for a subword of the cyclic word $\left(u_{r}\right)$. (The assertion for a subword of the cyclic word $\left(u_{r}^{-1}\right)$ follows from this and the facts that $S_{1}$ and $S_{2}$ are symmetric and that $w$ is a piece if and only if $w^{-1}$ is a piece.) To show the only if part, let $w$ be a piece which is a subword of $\left(u_{r}\right)$. Suppose on the contrary that $S(w)$ contains $S_{1}$ or $\left(\ell_{1}, S_{2}, \ell_{2}\right)\left(\ell_{1}, \ell_{2} \in \mathbb{Z}_{+}\right)$as a subsequence. Let $w^{\prime}$ be a subword of $w$ corresponding to the subsequence. Then, since each of $S_{1}$ and $S_{2}$ appears only twice in the cyclic sequence $C S(r)$ by Proposition $3.12(2)$, we have the following.

(i) If $S\left(w^{\prime}\right)=S_{1}$, then $w^{\prime}=v_{1}$ or $v_{3}$.

(ii) If $S\left(w^{\prime}\right)=\left(\ell_{1}, S_{2}, \ell_{2}\right)$, then $w^{\prime}=v_{1 e} v_{2} v_{3 b}$ or $v_{3 e} v_{4} v_{1 b}$.

In either case, $w^{\prime}$ cannot be a subword of any of the maximal 1-pieces of $\left(u_{r}\right)$ listed in Lemma 3.24(2a). Hence, $w$ is not a piece, a contradiction. To see the if part, let $w$ be a subword of $\left(u_{r}\right)$ whose $S$-sequence does not contain $S_{1}$ nor $\left(\ell_{1}, S_{2}, \ell_{2}\right)\left(\ell_{1}, \ell_{2} \in \mathbb{Z}_{+}\right)$as a subsequence. Then we see by using Proposition $3.12(2)$ that $w$ does not contain $v_{1}, v_{3}, v_{1 e} v_{2} v_{3 b}$, nor $v_{3 e} v_{4} v_{1 e}$ as a subword. Since $w$ is a subword of $\left(u_{r}\right)$, this implies that $w$ is a subword of one of the maximal 1-pieces of $\left(u_{r}\right)$ listed in Lemma 3.24(2a). Hence $w$ is a piece.

(2) As in (1), we prove the assertion for a subword of the cyclic word $\left(u_{r}\right)$. Suppose that $w$ is a subword of $\left(u_{r}\right)$ such that $S(w)$ contains either $\left(S_{1}, S_{2}, \ell\right)$ or $\left(\ell, S_{2}, S_{1}\right)$ with $\ell \in \mathbb{Z}_{+}$. Let $w^{\prime}$ be a subword of $w$ corresponding to the subsequence. Then, since $S_{1}$ and $S_{2}$ appears only twice in the cyclic sequence $C S(r)$ by Proposition $3.12(2)$, we have the following.

(i) If $S\left(w^{\prime}\right)=\left(S_{1}, S_{2}, \ell\right)$, then $w^{\prime}=v_{1} v_{2} v_{3 b}$ or $v_{3} v_{4} v_{1 b}$.

(ii) If $S\left(w^{\prime}\right)=\left(\ell, S_{2}, S_{1}\right)$, then $w^{\prime}=v_{1 e} v_{2} v_{3}$ or $v_{3 e} v_{4} v_{1}$.

In either case, $w^{\prime}$ cannot be a subword of any of the maximal 2-pieces of $\left(u_{r}\right)$ listed in Lemma $3.24(2 \mathrm{~b})$. Hence, $w$ is not a product of two pieces, as desired.

\section{Annular Diagrams OVER 2-BRIDGE LINK GROUPS}

In this section, we establish a very strong structure theorem (Theorems 4.9 and 4.11) for the annular diagram which arises in the study of the conjugacy problem by using the small cancellation theory. The structure theorem forms the cornerstone of this whole series of papers. 
Let us begin with necessary definitions and notation following [10]. A map $M$ is a finite 2-dimensional cell complex embedded in $\mathbb{R}^{2}$. To be precise, $M$ is a finite collection of vertices (0-cells), edges (1-cells), and faces (2-cells) in $\mathbb{R}^{2}$ satisfying the following conditions.

(i) A vertex is a point in $\mathbb{R}^{2}$.

(ii) An edge $e$ is homeomorphic to an open interval such that $\bar{e}=e \cup\{a\} \cup$ $\{b\}$, where $a$ and $b$ are vertices of $M$ which are possibly identical.

(iii) For each face $D$ of $M$, there is a continuous map $f$ from the 2-ball $B^{2}$ to $\mathbb{R}^{2}$ such that

(a) the restriction of $f$ to the interior of $B^{2}$ is a homeomorphism onto $D$, and

(b) the image of $\partial B^{2}$ is equal to $\cup_{i=1}^{k} \bar{e}_{i}$ for some set $\left\{e_{1}, \ldots, e_{k}\right\}$ of edges of $M$.

The underlying space of $M$, i.e., the union of the cells in $M$, is also denoted by the same symbol $M$. The boundary (frontier), $\partial M$, of $M$ in $\mathbb{R}^{2}$ is regarded as a 1-dimensional subcomplex of $M$. An edge may be traversed in either of two directions. If $v$ is a vertex of a map $M, d_{M}(v)$, the degree of $v$, will denote the number of oriented edges in $M$ having $v$ as initial vertex. A vertex $v$ of $M$ is called an interior vertex if $v \notin \partial M$, and an edge $e$ of $M$ is called an interior edge if $e \not \subset \partial M$.

A path in $M$ is a sequence of oriented edges $e_{1}, \ldots, e_{n}$ such that the initial vertex of $e_{i+1}$ is the terminal vertex of $e_{i}$ for every $1 \leq i \leq n-1$. A cycle is a closed path, namely a path $e_{1}, \ldots, e_{n}$ such that the initial vertex of $e_{1}$ is the terminal vertex of $e_{n}$. If $D$ is a face of $M$, any cycle of minimal length which includes all the edges of the boundary, $\partial D$, of $D$ going around once along the boundary of $D$ is called a boundary cycle of $D$. To be precise it is defined as follows. Let $f: B^{2} \rightarrow D$ be a continuous map satisfying the condition (iii) above. We may assume that $\partial B^{2}$ has a cellular structure such that the restriction of $f$ to each cell is a homeomorphism. Choose an arbitrary orientation of $\partial B^{2}$, and let $\hat{e}_{1}, \ldots, \hat{e}_{n}$ be the oriented edges of $\partial B^{2}$, which are oriented in accordance with the orientation of $\partial B^{2}$ and which lie on $\partial B^{2}$ in this cyclic order with respect to the orientation of $\partial B^{2}$. Let $e_{i}$ be the orientated edge $f\left(\hat{e}_{i}\right)$ of $M$. Then the cycle $e_{1}, \ldots, e_{n}$, is a boundary cycle of $D$.

Definition 4.1. A non-empty map $M$ is called a $[p, q]$-map if the following conditions hold.

(i) $d_{M}(v) \geq p$ for every interior vertex $v$ of $M$.

(ii) $d_{M}(D) \geq q$ for every face $D$ of $M$. 
Definition 4.2. Let $R$ be a symmetrized subset of the free group $F(X)$ with basis $X$. An $R$-diagram is a map $M$ and a function $\phi$ assigning to each oriented edge $e$ of $M$, as a label, a reduced word $\phi(e)$ in $X$ such that the following hold.

(i) If $e$ is an oriented edge of $M$ and $e^{-1}$ is the oppositely oriented edge, then $\phi\left(e^{-1}\right)=\phi(e)^{-1}$.

(ii) For any boundary cycle $\delta$ of any face of $M, \phi(\delta)$ is a cyclically reduced word representing an element of $R$. (If $\alpha=e_{1}, \ldots, e_{n}$ is a path in $M$, we define $\phi(\alpha) \equiv \phi\left(e_{1}\right) \cdots \phi\left(e_{n}\right)$.)

Let $D_{1}$ and $D_{2}$ be faces (not necessarily distinct) of $M$ with an edge $e \subseteq$ $\partial D_{1} \cap \partial D_{2}$. Let $e \delta_{1}$ and $\delta_{2} e^{-1}$ be boundary cycles of $D_{1}$ and $D_{2}$, respectively. Let $\phi\left(\delta_{1}\right)=f_{1}$ and $\phi\left(\delta_{2}\right)=f_{2}$. An $R$-diagram $M$ is said to be reduced if one never has $f_{2}=f_{1}^{-1}$. It should be noted that if $M$ is reduced then $\phi(e)$ is a piece for every interior edge $e$ of $M$.

Convention 4.3. [6, Convention 1] Let $R$ be the symmetrized subset of $F(a, b)$ generated by the single relator $u_{r}$ of the upper presentation of $G(K(r))$. For any $R$-diagram $M$, we assume that $M$ satisfies the following.

(1) $d_{M}(v) \geq 3$ for every interior vertex $v$ of $M$.

(2) For every edge $e$ of $\partial M$, the label $\phi(e)$ is a piece.

(3) For a path $e_{1}, \ldots, e_{n}$ in $\partial M$ of length $n \geq 2$ such that the vertex $\bar{e}_{i} \cap$ $\bar{e}_{i+1}$ has degree 2 for $i=1,2, \ldots, n-1, \phi\left(e_{1}\right) \phi\left(e_{2}\right) \cdots \phi\left(e_{n}\right)$ cannot be expressed as a product of fewer than $n$ pieces.

Indeed, we may assume (1), because if there are two interior edges $e_{1}$ and $e_{2}$ meeting in an interior vertex of degree two, then we can delete the vertex $v$ and unite $e_{1}$ and $e_{2}$ into a single edge $e$ with label $\phi(e)=\phi\left(e_{1}\right) \phi\left(e_{2}\right)$. To see (2), recall that the assumption that $M$ is reduced implies that $\phi(e)$ is a piece for every interior edge $e$ of $M$. On the other hand, since the cyclic word $\left(u_{r}\right)$ can be written as a product of pieces, we may also assume that $\phi(e)$ is a piece for every edge $e$ in $\partial M$. Finally, we may assume (3), because if $\phi\left(e_{1}\right) \cdots \phi\left(e_{n}\right)$ is expressed as a product of less than $n$ pieces, then we can change the cellular structure of the interval $e_{1} \cup \cdots \cup e_{n}$ so that the new cellular structure has fewer vertices compared with the original one.

The following corollary is immediate from Proposition 3.22 and Convention 4.3 .

Corollary 4.4. 6, Corollary 6.2] Suppose that $r$ is a rational number with $0<r<1$. Let $R$ be the symmetrized subset of $F(a, b)$ generated by the single relator $u_{r}$ of the upper presentation of $G(K(r))$. Then every reduced $R$-diagram is a [4,4]-map. 
We turn to interpreting conjugacy in terms of diagrams.

Definition 4.5. An annular map $M$ is a connected map such that $\mathbb{R}^{2}-M$ has exactly two connected components. It is said to be nontrivial if it contains 2-cells. For a symmetrized subset $R$ of $F(a, b)$, an annular $R$-diagram is an $R$-diagram whose underlying map is an annular map.

Let $M$ be an annular $R$-diagram, and let $K$ and $H$ be, respectively, the unbounded and bounded components of $\mathbb{R}^{2}-M$. We call $\partial K(\subset \partial M)$ the outer boundary of $M$, while $\partial H(\subset \partial M)$ is called the inner boundary of $M$. Clearly, the boundary of $M, \partial M$, is the union of the outer boundary and the inner boundary. A cycle of minimal length which contains all the edges in the outer (inner, resp.) boundary of $M$ going around once along the boundary of $K$ (H, resp.) is an outer (inner, resp.) boundary cycle of $M$. An outer (inner, resp.) boundary label of $M$ is defined to be a word $\phi(\alpha)$ in $X$ for $\alpha$ an outer (inner, resp.) boundary cycle of $M$.

Convention 4.6. Since $M$ is embedded in $\mathbb{R}^{2}$, each 2-cell of $M$ inherits an orientation of $\mathbb{R}^{2}$. Throughout this series of papers, we assume, unlike the usual orientation convention, that $\mathbb{R}^{2}$ is oriented so that the boundary cycles of the 2-cells of $M$ are clockwise. Thus the outer boundary cycles are clockwise and inner boundary cycles are counterclockwise, unlike the convention in [10, p.253].

The following lemma is a well-known classical result in combinatorial group theory.

Lemma 4.7. [10, Lemmas V.5.1 and V.5.2] Suppose $G=\langle X \mid R\rangle$ with $R$ being symmetrized. Let $u, v$ be two cyclically reduced words in $X$ which are not trivial in $G$ and which are not conjugate in $F(X)$. Then $u$ and $v$ represent conjugate elements in $G$ if and only if there exists a reduced nontrivial annular $R$-diagram $M$ such that $u$ is an outer boundary label and $v^{-1}$ is an inner boundary label of $M$.

The following lemma will play an essential role in the proof of Theorem 4.9 below.

Lemma 4.8. [10, Theorem V.3.1] Let $M$ be an arbitrary connected map. Then

$$
4-4 h \leq \sum_{v \in \partial M}\left(3-d_{M}(v)\right)+\sum_{v \in M-\partial M}\left(4-d_{M}(v)\right)+\sum_{D \in M}\left(4-d_{M}(D)\right),
$$


where $h$ is the number of holes of $M$, i.e., the number of bounded components of $\mathbb{R}^{2}-M$. In particular, if $M$ is a $[4,4]$-map, then

$$
4-4 h \leq \sum_{v \in \partial M}\left(3-d_{M}(v)\right)
$$

In the above lemma and throughout this paper, the symbol $v \in X(D \in X$, respectively) under the symbol $\sum$, where $X$ is a map $M$ or a subspace of a map $M$, means that the sum is over the vertices $v$ (the faces $D$, respectively) of the map $M$ contained in the subspace $X$.

Proof. We repeat the proof of a part of [10, Theorem V.3.1]. Put

$V=$ the number of vertices of $M$;

$E=$ the number of (unoriented) edges of $M$;

$F=$ the number of faces of $M$;

$V^{\bullet}=$ the number of vertices in $\partial M$;

$E^{\bullet}=$ the number of (unoriented) edges in $\partial M$, counted with multiplicity.

To be more precise, $E^{\bullet}$ means the number of (unoriented) edges on $\partial M$ with an edge counted twice if it appears twice in the cycles necessary to describe the boundary of $M$. For example, the edge $e_{0}$ in each of Figure 5(a) and Figure 5(b) has to be counted twice in computing $E^{\bullet}$, because $e_{0}$ in Figure 5)(a) occurs twice in a boundary cycle of $M$ and $e_{0}$ in Figure 5(b) occurs in both inner and outer boundary cycles of $M$. However the vertex $v_{0}$ in each of Figure 5(a) and Figure 5(b) has to be counted only once in computing $V^{\bullet}$.

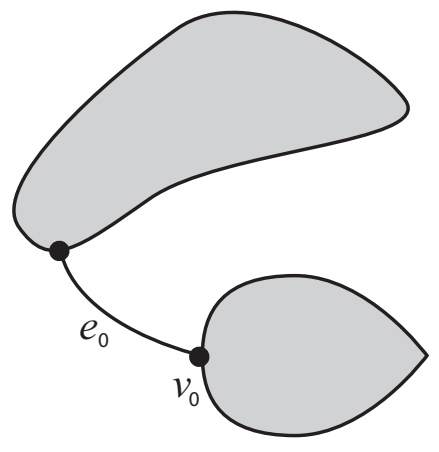

(a)

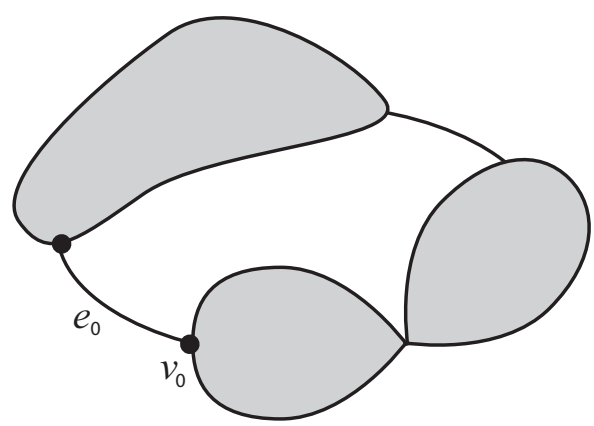

(b)

Figure 5. The edge $e_{0}$ is counted twice in computing $E^{\bullet}$, while the vertex $v_{0}$ is counted once in computing $V^{\bullet}$. 
Since $M$ has $h$ holes, it follows from Euler's Formula that

$$
1-h=V-E+F .
$$

Putting $d(v)=d_{M}(v)$ for vertices $v$ of $M$ and $d(D)=d_{M}(D)$ for faces $D$ of $M$, it is easy to observe that

$$
\begin{aligned}
& 2 E=\sum_{v \in M} d(v) ; \\
& 2 E=\sum_{D \in M} d(D)+E^{\bullet} .
\end{aligned}
$$

These equations together with (†) yield

$$
\begin{aligned}
4-4 h & =4 V+4 F-\sum_{v \in M} d(v)-\sum_{D \in M} d(D)-E^{\bullet} \\
& =\sum_{v \in M}(4-d(v))+\sum_{D \in M}(4-d(D))-E^{\bullet} \\
& =\sum_{v \in \partial M}(4-d(v))+\sum_{v \in M-\partial M}(4-d(v))+\sum_{D \in M}(4-d(D))-E^{\bullet} \\
& =\sum_{v \in \partial M}(3-d(v))+\sum_{v \in M-\partial M}(4-d(v))+\sum_{D \in M}(4-d(D))+V^{\bullet}-E^{\bullet} .
\end{aligned}
$$

Note that

$$
V^{\bullet}-E^{\bullet} \leq V^{\bullet}-E_{0}^{\bullet}=\chi(\partial M)=\beta_{0}(\partial M)-\beta_{1}(\partial M) \leq 0,
$$

where $E_{0}^{\bullet}$ is the number of (unoriented) edges in $\partial M$ (counted without multiplicity), $\chi$ denotes the Euler characteristic, and $\beta_{i}$ denotes the $i$-th Betti number. The last inequality follows from the fact that each component $C$ of $\partial M$ has a positive first Betti number, which in turn follows from the fact that $\mathbb{R}^{2}-C$ is not connected. Hence, the required result follows.

For a rational number $r$ with $0<r<1$, let $R$ be the symmetrized subset of $F(a, b)$ generated by the single relator $u_{r}$ of the upper presentation of $G(K(r))$. Our goal of the present section is to describe the structure of reduced annular $R$-diagrams with $u_{s}$ and $u_{s^{\prime}}^{ \pm 1}$, respectively, as outer and inner boundary labels, where $u_{s}$ and $u_{s^{\prime}}\left(s, s^{\prime} \in I_{1}(r) \cup I_{2}(r)\right)$ are the cyclically reduced words in $\{a, b\}$ obtained from the simple loops $\alpha_{s}$ and $\alpha_{s^{\prime}}$, respectively, as in Lemma 3.1. We obtain the following very strong structure theorem.

Theorem 4.9. (Structure Theorem) Suppose that $r$ is a rational number with $0<r<1$. Let $R$ be the symmetrized subset of $F(a, b)$ generated by the single 
relator $u_{r}$ of the upper presentation of $G(K(r))$, and let $S(r)=\left(S_{1}, S_{2}, S_{1}, S_{2}\right)$ be as in Proposition 3.12. Suppose that $M$ is a reduced nontrivial annular $R$-diagram such that, for $\alpha$ and $\delta$ which are, respectively, arbitrary outer and inner boundary cycles of $M$,

(i) the words $\phi(\alpha)$ and $\phi(\delta)$ are cyclically reduced;

(ii) the words $\phi(\alpha)$ and $\phi(\delta)$ are cyclically alternating;

(iii) the cyclic $S$-sequences of the cyclic words $(\phi(\alpha))$ and $(\phi(\delta))$ do not contain $\left(S_{1}, S_{2}\right)$ nor $\left(S_{2}, S_{1}\right)$ as a subsequence.

Let the outer and inner boundaries of $M$ be denoted by $\sigma$ and $\tau$, respectively. Then the following hold.

(1) The outer and inner boundaries $\sigma$ and $\tau$ are simple, i.e., they are homeomorphic to the circle, and there is no edge contained in $\sigma \cap \tau$.

(2) $d_{M}(v)=2$ or 4 for every vertex $v$ of $\partial M$. Moreover, on both $\sigma$ and $\tau$, vertices of degree 2 appear alternately with vertices of degree 4.

(3) $d_{M}(v)=4$ for every interior vertex $v$ of $M$.

(4) $d_{M}(D)=4$ for every face $D$ of $M$.

Before proving the theorem, we prepare the following lemma.

Lemma 4.10. Under the assumption of Theorem 4.9, the following hold.

(1) There is no face $D$ in $M$ such that $\partial D \cap \partial M$ contains three edges $e_{1}, e_{2}$ and $e_{3}$ such that $\bar{e}_{1} \cap \bar{e}_{2}=\left\{v_{1}\right\}$ and $\bar{e}_{2} \cap \bar{e}_{3}=\left\{v_{2}\right\}$, where $d_{M}\left(v_{i}\right)=2$ for each $i=1,2$.

(2) The outer and inner boundaries $\sigma$ and $\tau$ are simple.

Proof. (1) Suppose on the contrary that there is a face $D$ in $M$ and edges $e_{1}$, $e_{2}$ and $e_{3}$ in $\partial D \cap \partial M$ satisfying the condition. Then $e_{1}, e_{2}$ and $e_{3}$ form three consecutive edges in the outer or inner boundary cycle, say the outer boundary cycle $\alpha$. By Convention 4.3(2)-(3), the product $\phi\left(e_{1}\right) \phi\left(e_{2}\right) \phi\left(e_{3}\right)$ which is a subword of the cyclic word $\left(u_{r}^{ \pm 1}\right)$ cannot be expressed as a product of less than 3 pieces. Because of the symmetry of $S_{1}$ and $S_{2}$ (Proposition $3.12(1)$ ) and Lemma 3.5. we may assume without loss of generality that $\phi\left(e_{1}\right) \phi\left(e_{2}\right) \phi\left(e_{3}\right)$ is a subword of the cyclic word $\left(u_{r}\right)$. We also assume that the length $k$ of the continued fraction $r=\left[m_{1}, m_{2}, \ldots, m_{k}\right]$ is greater than 1 . (The proof for the case $k=1$ is analogous to the proof for the general case $k \geq 2$.) Let $w_{0}$ be the maximal 2-piece which forms a proper initial subword of $\phi\left(e_{1}\right) \phi\left(e_{2}\right) \phi\left(e_{3}\right)$. Then $w_{0}$ is equal to one of the words in Lemma 3.24(2b). If $w_{0}$ is equal to $v_{1} v_{2}$ or $v_{1 e} v_{2} v_{3 b}^{*}$, then $\phi\left(e_{1}\right) \phi\left(e_{2}\right) \phi\left(e_{3}\right)$ contains a subword $w$ such that the $S$ sequence of $w$ is $\left(S_{1}, S_{2}, \ell\right)$ or $\left(\ell, S_{2}, S_{1}\right)$ accordingly, for some positive integer $\ell$. By Proposition 3.12, this implies that the cyclic $S$-sequences of one of the 
cyclic words $(\phi(\alpha))$ and $(\phi(\delta))$ contains $\left(S_{1}, S_{2}\right)$ or $\left(S_{2}, S_{1}\right)$ as a subsequence. This contradicts the hypothesis (iii). The remaining possibilities for $w_{0}$ can be treated similarly.

(2) Suppose on the contrary that $\sigma$ or $\tau$ is not simple. Then there is an extremal disk, say $J$, which is properly contained in $M$ and connected to the rest of $M$ by a single vertex. Here, recall that an extremal disk of a map $M$ is a submap of $M$ which is topologically a disk and which has a boundary cycle $e_{1}, \ldots, e_{n}$ such that the edges $e_{1}, \ldots, e_{n}$ occur in order in some boundary cycle of the whole map $M$. Clearly $J$ is a connected and simply connected map having at least one face. Furthermore, by Corollary 4.4, $J$ is a [4, 4]-map. Then by Lemma 4.8, we have

$$
\sum_{v \in \partial J}\left(3-d_{J}(v)\right) \geq 4
$$

Putting

$$
A=\left\{v \in \partial J \mid d_{J}(v)=2\right\} \quad \text { and } \quad B=\left\{v \in \partial J \mid d_{J}(v) \geq 4\right\},
$$

it is easy to see that $A$ has at least 4 more elements than $B$ does in order to satisfy inequality ( $\$$. Since $J$ is connected to the rest of $M$ by a single vertex, say $v_{0}$, then every vertex in $\partial J$ except $v_{0}$ belongs to either $A$ or $B$ and $d_{J}\left(v_{0}\right)=d_{M}\left(v_{0}\right)-1 \geq 3$ (note that $d_{M}\left(v_{0}\right) \geq 4$, since $v_{0} \in \partial M$ ). So there are at least 2 adjacent vertices, say $v_{1}$ and $v_{2}$, belonging to $A$. But then there is a face $D$ in $J$, so in $M$, such that $\partial D \cap \partial M$ contains three consecutive edges $e_{1}, e_{2}$ and $e_{3}$ such that $\bar{e}_{1} \cap \bar{e}_{2}=\left\{v_{1}\right\}$ and $\bar{e}_{2} \cap \bar{e}_{3}=\left\{v_{2}\right\}$, where $d_{M}\left(v_{i}\right)=d_{J}\left(v_{i}\right)=2$ for each $i=1,2$, contradicting Lemma 4.10(1).

Proof. of Theorem 4.9 Note first that $M$ is a connected annular [4, 4]-map by Corollary 4.4. By hypothesis (i), there is no vertex of degree 1 in $\partial M$. Moreover, there is no vertex of degree 3 in $\partial M$, as is shown in the following. Suppose there is a vertex $v \in \partial M$ of degree 3 . Then there are at most two faces which contain $v$. If there are two such faces, then one of $\phi(\alpha)$ and $\phi(\delta)$ is not cyclically alternating, a contradiction to hypothesis (ii). If there is a unique such face, then by using hypothesis (ii), we see that the boundary label of the face is not cyclically alternating, a contradiction. Finally, if there is no such face, then this contradicts hypothesis (ii). Hence, no vertex in $\partial M$ has degree 1 nor 3, and so every vertex in $\partial M$ must have degree 2 or at least 4 .

We argue two cases separately.

Case 1. $\sigma \cap \tau=\emptyset$.

In this case, (1) follows immediately from Lemma 4.10(2). 
(2) Since $\partial M$ is the disjoint union of $\sigma$ and $\tau$, Lemma 4.8 yields

$$
0 \leq \sum_{v \in \sigma}\left(3-d_{M}(v)\right)+\sum_{v \in \tau}\left(3-d_{M}(v)\right) .
$$

On the other hand, since $\sigma$ and $\tau$ are simple by Lemma 4.10(2) and since they are disjoint by the current assumption, every edge in $\sigma$ or $\tau$ is contained in the boundary of a unique face of $M$. Thus Lemma 4.10(1) implies that vertices of degree 2 do not occur consecutively on $\sigma$ nor on $\tau$. Hence, the above inequality holds only when $d_{M}(v)=2$ or $d_{M}(v)=4$ for every vertex $v \in \sigma \cup \tau$ and when vertices of degree 2 appear alternately with vertices of degree 4 on both $\sigma$ and $\tau$, thus proving (2).

(3)-(4) By (2), $\sum_{v \in \partial M}\left(3-d_{M}(v)\right)=0$. This together with Lemma 4.8 yields

$$
0 \leq \sum_{v \in M-\partial M}\left(4-d_{M}(v)\right)+\sum_{D \in M}\left(4-d_{M}(D)\right) .
$$

Here, since $4-d_{M}(v) \leq 0$ for every $v \in M-\partial M$ and $4-d_{M}(D) \leq 0$ for every $D \in M$ by the definition of a [4,4]-map, the only possibility is that $4-d_{M}(v)=0$ for every vertex $v \in M-\partial M$ and $4-d_{M}(D)=0$ for every face $D \in M$, thus proving (3) and (4).

Case 2. $\sigma \cap \tau \neq \emptyset$.

(1) Suppose on the contrary that $\sigma \cap \tau$ contains an edge. As illustrated in Figure [6, there is a submap $J$ of $M$ such that

(i) $J$ is bounded by a simple closed path of the form $\sigma_{1} \tau_{1}$, where $\sigma_{1} \subseteq \sigma$ and $\tau_{1} \subseteq \tau$

(ii) $J$ is connected to the rest of $M$ by two distinct vertices, say $v_{1}$ and $v_{2}$, where $\sigma_{1} \cap \tau_{1}=\left\{v_{1}, v_{2}\right\}$ and $v_{1}$ is an endpoint of an edge contained in $\sigma \cap \tau$. Note that $d_{J}\left(v_{1}\right)=d_{M}\left(v_{1}\right)-1 \geq 3$ and $d_{J}\left(v_{2}\right) \geq 2$.

Since $J$ is a connected and simply connected [4,4]-map, Lemma 4.8 yields

$$
4 \leq \sum_{v \in \partial J}\left(3-d_{J}(v)\right) .
$$

Since $d_{J}\left(v_{1}\right) \geq 3$ and $d_{J}\left(v_{2}\right) \geq 2$, this inequality implies

$$
3 \leq \sum_{v \in \partial J-\left\{v_{1}, v_{2}\right\}}\left(3-d_{J}(v)\right) .
$$

On the other hand, since every vertex in $\partial J-\left\{v_{1}, v_{2}\right\}$ has degree 2 or at least 4 and since degree 2 vertices cannot occur consecutively on $\sigma_{1}-\left\{v_{1}, v_{2}\right\}$ nor 
on $\tau_{1}-\left\{v_{1}, v_{2}\right\}$ (see Lemma 4.10(1)),

$$
\sum_{v \in \partial J-\left\{v_{1}, v_{2}\right\}}\left(3-d_{J}(v)\right) \leq 2,
$$

a contradiction.

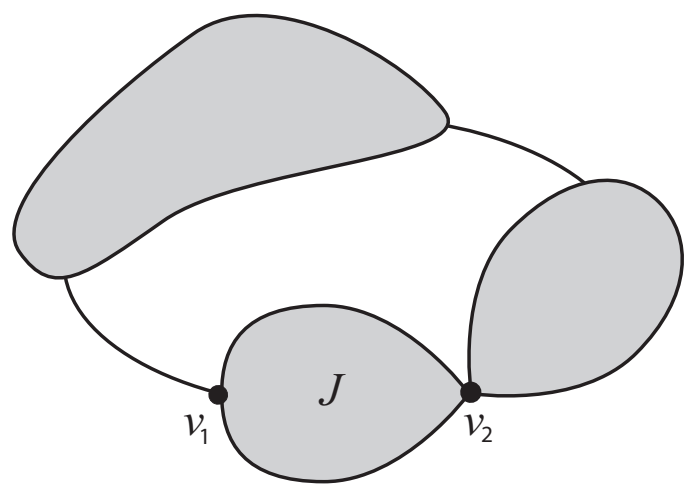

Figure 6. A possible annular map $M$ when $\sigma \cap \tau$ contains an edge.

(2)-(4) By (1), $\sigma \cap \tau$ consists of finitely many vertices in $M$. Then, by Lemma 4.10(1), vertices of degree 2 do not occur consecutively on $\sigma$ nor on $\tau$.

First suppose that $\sigma \cap \tau$ consists of at least two vertices, say $v_{1}, \ldots, v_{n}$, where $n \geq 2$ and where these vertices are indexed according as there is a submap $J_{i}$ of $M$ for every $i=1, \ldots, n$ such that

(i) $J_{i}$ is bounded by a simple closed path of the form $\sigma_{i} \tau_{i}$, where $\sigma_{i} \subseteq \sigma$ and $\tau_{i} \subseteq \tau$

(ii) $J_{i}$ is connected to the rest of $M$ by two distinct vertices, say $v_{i}$ and $v_{i+1}$, where $\sigma_{i} \cap \tau_{i}=\left\{v_{i}, v_{i+1}\right\}$ and where $d_{J_{i}}\left(v_{i}\right), d_{J_{i}}\left(v_{i+1}\right) \geq 2$ and $d_{J_{i}}\left(v_{i+1}\right)+d_{J_{i+1}}\left(v_{i+1}\right)=d_{M}\left(v_{i+1}\right)$ (taking the indices modulo $n$ ).

Then each $J_{i}$ is a connected and simply connected [4,4]-map such that $M=$ $J_{1} \cup \cdots \cup J_{n}$. Moreover $\sigma=\sigma_{1} \cup \cdots \cup \sigma_{n}$ and $\tau=\tau_{1} \cup \cdots \cup \tau_{n}$. The same argument as for $\left(M^{\prime}, v_{0}^{\prime}, v_{0}^{\prime \prime}\right)$ above applies to each $\left(J_{i}, v_{i}, v_{i+1}\right)$ to prove the assertions.

Next suppose that $\sigma \cap \tau$ consists of a single vertex, say $v_{0}$. Cut $M$ open at $v_{0}$ to get a connected and simply connected [4,4]-map $M^{\prime}$. In this process, the vertex $v_{0}$ is separated into two distinct vertices, say $v_{0}^{\prime}$ and $v_{0}^{\prime \prime}$, in $M^{\prime}$ such that $d_{M^{\prime}}\left(v_{0}^{\prime}\right), d_{M^{\prime}}\left(v_{0}^{\prime \prime}\right) \geq 2$ and $d_{M^{\prime}}\left(v_{0}^{\prime}\right)+d_{M^{\prime}}\left(v_{0}^{\prime \prime}\right)=d_{M}\left(v_{0}\right)$. Then $M^{\prime}$ is bounded by a simple closed path of the form $\sigma_{0} \tau_{0}$, where $\sigma_{0} \cap \tau_{0}=\left\{v_{0}^{\prime}, v_{0}^{\prime \prime}\right\}$. Again by 
Lemma 4.8,

$$
4 \leq \sum_{v \in \partial M^{\prime}}\left(3-d_{M^{\prime}}(v)\right)
$$

Considering that vertices of degree 2 do not occur consecutively on $\sigma_{0}-\left\{v_{0}^{\prime}, v_{0}^{\prime \prime}\right\}$ nor on $\tau_{0}-\left\{v_{0}^{\prime}, v_{0}^{\prime \prime}\right\}$ (see Lemma 4.10(1)), we see that only the equality can hold, and that the equality holds only when $d_{M^{\prime}}(v)=2$ or 4 for every vertex $v \in \sigma_{0} \cup \tau_{0}-\left\{v_{0}^{\prime}, v_{0}^{\prime \prime}\right\}, d_{M^{\prime}}\left(v_{0}^{\prime}\right)=2=d_{M^{\prime}}\left(v_{0}^{\prime \prime}\right)$ and when vertices of degree 2 appear alternately with vertices of degree 4 starting and ending with vertices of degree 2 on both $\sigma_{0}-\left\{v_{0}^{\prime}, v_{0}^{\prime \prime}\right\}$ and $\tau_{0}-\left\{v_{0}^{\prime}, v_{0}^{\prime \prime}\right\}$. This implies that $d_{M}(v)=2$ or 4 for every vertex $v \in \partial M-\left\{v_{0}\right\}, d_{M}\left(v_{0}\right)=4$, and that vertices of degree 2 appear alternately with vertices of degree 4 on both $\sigma$ and $\tau$, thus proving (2).

Since $\sum_{v \in \partial M^{\prime}}\left(3-d_{M^{\prime}}(v)\right)=4$, Lemma 4.8 yields

$$
0 \leq \sum_{v \in M^{\prime}-\partial M^{\prime}}\left(4-d_{M^{\prime}}(v)\right)+\sum_{D \in M^{\prime}}\left(4-d_{M^{\prime}}(D)\right) .
$$

Here, by the definition of a [4,4]-map, $4-d_{M^{\prime}}(v) \leq 0$ for every vertex $v \in$ $M^{\prime}-\partial M^{\prime}$ and $4-d_{M^{\prime}}(D) \leq 0$ for every face $D \in M^{\prime}$, we must have $d_{M}(v)=$ $d_{M^{\prime}}(v)=4$ for every vertex $v \in M^{\prime}-\partial M^{\prime}$ and $d_{M}(D)=d_{M^{\prime}}(D)=4$ for every face $D \in M^{\prime}$, thus proving (3) and (4).

Theorem 4.9 enables us to identify all possible shapes of the annular maps $M$. To describe the result, we define the outer boundary layer of an annular map $M$ to be the submap of $M$ consisting of all faces $D$ such that the intersection of $\partial D$ with the outer boundary of $M$ contains an edge, together with the edges and vertices contained in $\partial D$.

Theorem 4.11. Let $M$ be a reduced annular [4,4]-map satisfying the assumptions of Theorem 4.9. Then Figure $7(a)$ illustrates the only possible type of the outer boundary layer of $M$, while Figure $7(b)$ illustrates the only possible type of whole $M$. (All layers have the same number of faces, but the number may vary, as may the number of layers.)

Proof. Let $v_{1}, v_{2}, \ldots, v_{2 n}$ be the vertices of the outer boundary $\sigma$ arranged in this cyclic order in $\sigma$, such that $d_{M}\left(v_{i}\right)$ is 4 or 2 according to whether $i$ is odd or even (see Theorem 4.9(1) and (2)). Let $e_{i}(1 \leq i \leq 2 n)$ be the oriented edge in $\sigma$ running from $v_{i}$ to $v_{i+1}$, where the indices are taken modulo $2 n$. Then, for each $j(1 \leq j \leq n)$, there is a unique face, $D_{j}$, of $M$ whose boundary contains the vertices $v_{2 j-1}, v_{2 j}$ and $v_{2 j+1}$ and the edges $e_{2 j-1}$ and $e_{2 j}$. Since $d_{M}\left(D_{j}\right)=4$ by Theorem 4.9 (4), there are oriented edges $e_{2 j-1}^{\prime}$ and $e_{2 j}^{\prime}$ and of $M$ such that 


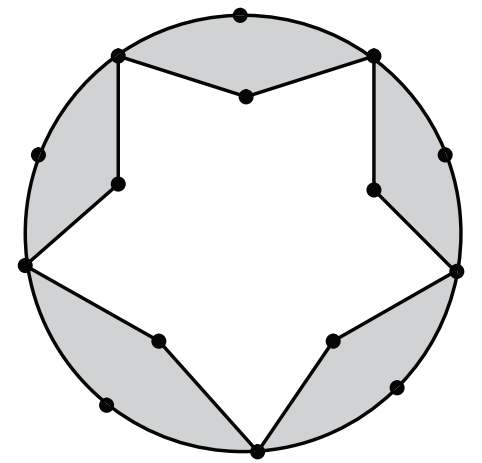

(a)

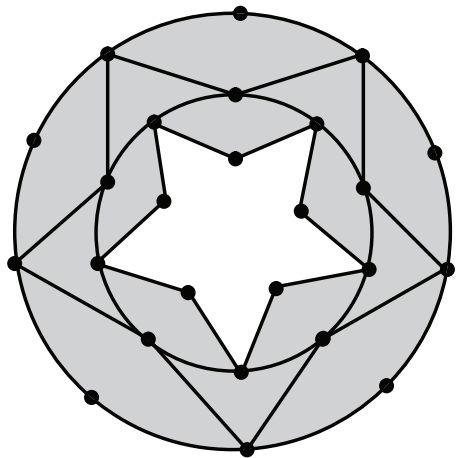

(b)

FIGURE 7.

$e_{2 j-1}, e_{2 j}, e_{2 j}^{-1}, e_{2 j-1}^{\prime-1}$ is a boundary cycle of $D_{j}$. The terminal point of $e_{2 j-1}^{\prime}$ is equal to the initial point of $e_{2 j}^{\prime}$, and we denote it by $v_{2 j}^{\prime}$ (see Figure 8).

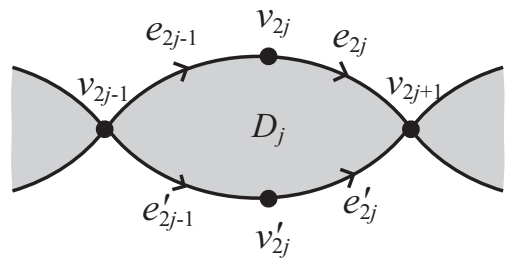

FIGURE 8.

Claim 1. The unoriented edges determined by $e_{i}^{\prime}$ and $e_{i+1}^{\prime}$ are distinct for every $1 \leq i \leq 2 n$, where indices are taken modulo $2 n$.

Proof. of Claim 1 Suppose this is not the case. Then, since $M$ is planar, we have $e_{i}^{\prime}=e_{i+1}^{\prime-1}$ for some $i$. Suppose first that $e_{2 j}^{\prime}=e_{2 j+1}^{\prime-1}$ for some $j$. Then the union of the closures $\bar{D}_{j}$ and $\bar{D}_{j+1}$ forms a neighborhood of $v_{2 j+1}$ in $M$ and hence, $e_{2 j}, e_{2 j+1}^{-1}$ and $e_{2 j}^{\prime}=e_{2 j+1}^{\prime-1}$ are the only oriented edges of $M$ having $v_{2 j+1}$ as the terminal point. So $d_{M}\left(v_{2 j+1}\right)=3$, a contradiction to Theorem 4.9)(2). Next, suppose $e_{2 j-1}^{\prime}=e_{2 j}^{\prime-1}$ for some $j$. Then $v_{2 j}^{\prime}$ is contained in the interior of the closure $\bar{D}_{j}$, and $e_{2 j-1}^{\prime}=e_{2 j}^{\prime-1}$ is the only oriented edge of $M$ having $v_{2 j}^{\prime}$ as the terminal point. So $d_{M}\left(v_{2 j}^{\prime}\right)=1$, a contradiction to Theorem 4.9 (3). 
Claim 2. For every $1 \leq j \leq n$, the oriented edges $e_{2 j}, e_{2 j+1}^{-1}, e_{2 j}^{\prime}, e_{2 j+1}^{\prime-1}$ are mutually distinct, and they are the only oriented edges of $M$ which have $v_{2 j+1}$ as the terminal point.

Proof. of Claim 2 Let $U$ be a small regular neighborhood of $v_{2 j+1}$ relative to $M$, namely, $U$ is a closed disk in $\mathbb{R}^{2}$ containing $v_{2 j+1}$ in its interior, such that $\left|M^{(1)}\right| \cap U$ consists of $4\left(=d_{M}\left(v_{2 j+1}\right)\right)$ arcs joining $v_{2 j+1}$ with $\partial U$, each pair of which intersect only at $v_{2 j+1}$. Here $\left|M^{(1)}\right|$ denotes the 1-skeleton of $M$, i.e., the union of the vertices and edges of $M$. Consider the punctured disk $\check{U}:=U-\left\{v_{2 j+1}\right\}$. Then the subsets $\left(e_{2 j} \cup e_{2 j+1}\right) \cap \check{U}$ and $\left(e_{2 j}^{\prime} \cup e_{2 j+1}^{\prime}\right) \cap \check{U}$ of $\check{U}$ are separated in $\check{U}$ by the union of open 2-cells $D_{j} \cup D_{j+1}$. Hence each of the oriented edges $e_{2 j}^{\prime}$ and $e_{2 j+1}^{\prime-1}$ cannot be identical with any of the oriented edges $e_{2 j}$ and $e_{2 j+1}^{-1}$. Since $e_{2 j} \neq e_{2 j+1}^{-1}$ by Theorem 4.9)(1) and $e_{2 j}^{\prime} \neq e_{2 j+1}^{\prime-1}$ by Claim 1, the oriented edges $e_{2 j}, e_{2 j+1}^{-1}, e_{2 j}^{\prime}, e_{2 j+1}^{-1}$ are mutually distinct. Since $d_{M}\left(v_{2 j+1}\right)=4$, we obtain the claim.

Claim 3. $e_{i}^{\prime}$ is not contained in $\sigma$ for every $1 \leq i \leq 2 n$.

Proof. of Claim 3 We prove the claim when $i=2 j$ for some integer $j$. (The remaining case can be treated by a parallel argument.) Suppose $e_{2 j}^{\prime}$ is contained in $\sigma$. Then, since $v_{2 j+1}$ is the terminal point of $e_{2 j}^{\prime}$, the oriented edge $e_{2 j}^{\prime}$ is equal to $e_{2 j}$ or $e_{2 j+1}^{-1}$. But, this is impossible by Claim 2.

Claim 4. $v_{2 j}^{\prime}$ is not contained in $\sigma$ for every $1 \leq j \leq n$.

Proof. of Claim 4 Suppose $v_{2 j}^{\prime}$ is contained in $\sigma$ for some $j$. Then $v_{2 j}^{\prime}=v_{k}$ for some $1 \leq k \leq 2 n$, and the oriented edges $e_{k-1}, e_{k}^{-1}, e_{2 j-1}^{\prime}, e_{2 j}^{\prime-1}$ have $v_{2 j}^{\prime}=v_{k}$ as the terminal point. Since these are mutually distinct by Claims 1 and 3, we have $d_{M}\left(v_{k}\right) \geq 4$ and hence $k=2 h+1$ for some integer $h$. If $h=j$, then $e_{2 j}^{\prime}$ is a loop based on $v_{2 j}^{\prime}=v_{2 j+1}$ and hence the oriented edge $e_{2 j}^{\prime-1}$ also has $v_{2 j+1}$ as the terminal point, a contradiction to Claim 2. Similarly, we see $h=j-1$ cannot happen. Hence we have $v_{2 j}^{\prime}=v_{2 h+1}$, where $h \neq j, j-1$.

Note that in addition to $e_{2 h}, e_{2 h+1}^{-1}, e_{2 h}^{\prime}, e_{2 h+1}^{-1}$, the oriented edges $e_{2 j-1}^{\prime}$ and $e_{2 j}^{\prime-1}$ have $v_{2 j}^{\prime}=v_{2 h+1}$ as the terminal point. By Claims 1 and 3, this implies that the pair $\left(e_{2 j-1}^{\prime}, e_{2 j}^{\prime-1}\right)$ is equal to $\left(e_{2 h}^{\prime}, e_{2 h+1}^{\prime-1}\right)$ or $\left(e_{2 h+1}^{\prime-1}, e_{2 h}^{\prime}\right)$. Since $M$ is planar, $\left(e_{2 j-1}^{\prime}, e_{2 j}^{\prime-1}\right)$ cannot be equal to $\left(e_{2 h}^{\prime}, e_{2 h+1}^{\prime-1}\right)$. So we may assume $\left(e_{2 j-1}^{\prime}, e_{2 j}^{\prime-1}\right)=\left(e_{2 h+1}^{\prime-1}, e_{2 h}^{\prime}\right)$. Then the initial point $v_{2 j+1}$ of $e_{2 j}^{\prime-1}$ is equal to the initial point $v_{2 h}^{\prime}$ of $e_{2 h}^{\prime}$. Similarly, we have $v_{2 j-1}=v_{2 h+2}^{\prime}$. Thus we have shown that the identity $v_{2 j}^{\prime}=v_{2 h+1}$ implies the identities $v_{2 h}^{\prime}=v_{2 j+1}$ and 
$v_{2 h+2}^{\prime}=v_{2 j-1}$. By repeatedly applying this fact, we see that, for every integer $k, v_{2(h-k)}^{\prime}=v_{2(j+k)+1}$ and $v_{2(j+k)}^{\prime}=v_{2(h-k)+1}$. Thus we can find a pair of integers $j^{*}$ and $h^{*}$ such that $v_{2 j^{*}}^{\prime}=v_{2 h^{*}+1}$ and $h^{*}=j^{*}$ or $j^{*}-1$. However, this is impossible by the argument in the first paragraph of this proof.

Claim 5. The unoriented edges determined by $e_{i}^{\prime}(1 \leq i \leq 2 n)$ are mutually distinct.

Proof. of Claim 5 Suppose this is not the case. Then, since $M$ is planar, we have $e_{i}^{\prime}=e_{k}^{\prime-1}$ for some $1 \leq i<k \leq 2 n$. We assume $i=2 j$ for some integer $j$. (The other case is treated by parallel arguments.) If $k=2 h$ for some integer $h$, then $e_{2 j}^{\prime}=e_{2 h}^{\prime-1}$, and hence the initial point $v_{2 j}^{\prime}$ of $e_{2 j}^{\prime}$ is equal to the initial point $v_{2 h+1}$ of $e_{2 h}^{\prime-1}$. This contradicts Claim 4. If $k=2 h+1$ for some integer $h$, then $e_{2 j}^{\prime}=e_{2 h+1}^{\prime-1}$, and hence the terminal point $v_{2 j+1}$ of $e_{2 j}^{\prime}$ is equal to the terminal point $v_{2 h+1}$ of $e_{2 h+1}^{\prime-1}$. Since $\sigma$ is simple, this implies $j=h$ and hence $e_{2 j}^{\prime}=e_{2 j+1}^{\prime-1}$. This contradicts Claim 1 .

Claim 6. The vertices $v_{2 j}^{\prime}(1 \leq j \leq n)$ are mutually distinct.

Proof. of Claim 6 Suppose $v_{2 j}^{\prime}=v_{2 h}^{\prime}$ for some $1 \leq j \neq h \leq n$. Then $e_{2 j-1}^{\prime}, e_{2 j}^{\prime-1}, e_{2 h-1}^{\prime}, e_{2 h}^{-1}$ have $v_{2 j}^{\prime}=v_{2 h}^{\prime}$ as the terminal point. Since these oriented edges are mutually distinct by Claim 5 and since $d_{M}\left(v_{2 j}^{\prime}\right)=2$ or 4 by Theorem $4.9(2),(3)$, we see that $d_{M}\left(v_{2 j}^{\prime}\right)=4$ and that these are the only oriented edges having $v_{2 j}^{\prime}=v_{2 h}^{\prime}$ as the terminal point. We can choose $j$ and $h$, so that they are outermost in the sense that $1 \leq j<h \leq n$ (after a cyclic permutation of indices) and that the vertices $v_{2 j}^{\prime}=v_{2 h}^{\prime}$ and $v_{2 k}^{\prime}(j<k<h)$ are mutually distinct. Then $\cup_{2 j \leq i \leq 2 h-1} \bar{e}_{i}^{\prime}$ is a simple loop. We show that $e_{2 j}^{\prime}$, $e_{2 h-1}^{\prime}$ are contained in the boundary of the annular diagram $M$. Suppose this is not the case. Then, by the above observations, there is a face, $D$, of $M$ whose boundary contains $e_{2 j}^{\prime}, e_{2 h-1}^{\prime}$. By Claim 2, the edges $e_{2 j+1}^{\prime}$ and $e_{2 h-2}^{\prime}$ must be contained in $\partial D$. Since $d_{M}(D)=4$ by Theorem $4.9(4)$, a boundary cycle of $D$ is given by $e_{2 j}^{\prime}, e_{2 j+1}^{\prime}, e_{2 h-2}^{\prime}, e_{2 h-1}^{\prime}$. So, $h=j+2$ and $v_{2 j+2}^{\prime}$ is contained in the interior of $\bar{D} \cup \bar{D}_{j+1}$. This implies that $v_{2 j+2}^{\prime}$ is an inner vertex and $d_{M}\left(v_{2 j+2}^{\prime}\right)=2$, a contradiction to Theorem 4.9 (3). Hence $e_{2 j}^{\prime}, e_{2 h-1}^{\prime}$ are contained in the boundary of the annular diagram $M$, and in particular, $v_{2 h-1}, v_{2 h}^{\prime}=v_{2 j}^{\prime}, v_{2 j+1}$ lie in the inner boundary of $M$ successively. However all of them have degree 4 . This contradicts Theorem 4.9)(2).

By Claims 5 and 6 , we see that $\sigma^{\prime}:=\cup_{i=1}^{2 n} \bar{e}_{i}^{\prime}$ is a simple loop and that $\sigma \cap \sigma^{\prime}=\left\{v_{2 j+1} \mid 0 \leq j \leq n-1\right\}$. Thus the outer boundary layer, $J$, of 
$M$ is as illustrated in Figure 7(a). If $J=M$, we obtain the conclusion of Theorem 4.11. So, in the following, we assume $J \varsubsetneqq M$. Let $M^{\prime}$ be the map obtained from $M$ by collapsing the outer boundary layer $J$ onto $\sigma^{\prime}$. Then $M^{\prime}$ is an annular map whose outer and inner boundaries are the simple loops $\sigma^{\prime}$ and $\tau$, respectively.

Claim 7. The annular map $M^{\prime}$ satisfies the conclusions of Theorem 4.9. Moreover, for each $1 \leq j \leq n$, there is a face $D_{j}^{\prime}$ of $M^{\prime}$ such that $e_{2 j}^{\prime}$ and $e_{2 j+1}^{\prime}$ are the only edges of $\sigma^{\prime}$ contained in $\partial D_{j}^{\prime}$.

Proof. of Claim 7 Since $J \varsubsetneqq M$, we may assume, after cyclic permutation of indices, that there is a face $D_{1}^{\prime}$ of $M^{\prime}$ whose boundary contains either $e_{2}^{\prime}$ or $e_{3}^{\prime}$. Then $\partial D_{1}^{\prime}$ contains both $e_{2}^{\prime}$ and $e_{3}^{\prime}$, because $d_{M}\left(v_{3}\right)=4$. By using the fact that $d_{M}\left(v_{1}\right)=d_{M}\left(v_{5}\right)=4$, we see that $e_{2}^{\prime}$ and $e_{3}^{\prime}$ are the only edges of $\sigma^{\prime}$ contained in $\partial D_{1}^{\prime}$. Since $d_{M}\left(D_{1}^{\prime}\right)=4$, there are oriented edges $e_{2}^{\prime \prime}$ and $e_{3}^{\prime \prime}$ of $M$ such that a boundary cycle of $D_{1}^{\prime}$ is $e_{2}^{\prime}, e_{3}^{\prime}, e_{3}^{\prime \prime-1}, e_{2}^{\prime \prime-1}$. This implies that $d_{M}\left(v_{4}^{\prime}\right) \geq 3$ and so $d_{M}\left(v_{4}^{\prime}\right)=4$. Thus there is an oriented edge, $e_{4}^{\prime \prime}$, emanating from $v_{4}^{\prime}$ other than $e_{3}^{\prime-1}, e_{3}^{\prime \prime-1}, e_{4}^{\prime}$. Since $d_{M}\left(v_{4}^{\prime}\right)=d_{M}\left(v_{5}\right)=4$, we see by Theorem 4.9(2) that the edge $e_{4}^{\prime}$ is not contained in the boundary of $M$. Hence, there is a face, $D_{2}^{\prime}$, of $M$ whose boundary contains both $e_{4}^{\prime}$ and $e_{4}^{\prime \prime}$. We see, by repeating the preceding argument for $D_{1}^{\prime}$, that $e_{5}^{\prime}$ is also contained in $\partial D_{2}^{\prime}$ and that $e_{4}^{\prime}$ and $e_{5}^{\prime}$ are the only edges of $\sigma^{\prime}$ contained in $\partial D_{2}^{\prime}$. Here, observe that $D_{1}^{\prime}=D_{2}^{\prime}$ if $n=1$. By repeating this argument, we see that, for each $1 \leq j \leq n$, there is a face $D_{j}^{\prime}$ of $M^{\prime}$ such that $e_{2 j}^{\prime}$ and $e_{2 j+1}^{\prime}$ are the only edges of $\sigma^{\prime}$ contained in $\partial D_{j}^{\prime}$. Hence the outer boundary $\sigma^{\prime}$ and the inner boundary $\tau$ of $M^{\prime}$ are disjoint. So $M^{\prime}$ satisfies the conclusion (1) of Theorem 4.9, The above fact also implies $d_{M^{\prime}}\left(v_{2 j}^{\prime}\right)=4$ and $d_{M^{\prime}}\left(v_{2 j+1}\right)=2$ for every $j$. Thus the outer boundary $\sigma^{\prime}$ of $M^{\prime}$ satisfies the conclusion (2). Since $d_{M^{\prime}}(v)=d_{M}(v)$ for any vertex of $M^{\prime}$ which is not contained in $\sigma$ and since $\tau$ is disjoint from $\sigma$, the inner boundary $\tau$ of $M^{\prime}$ also satisfies the conclusion (2) and $M^{\prime}$ satisfies the conclusion (3). It is obvious that $M^{\prime}$ satisfies the conclusion (4).

Note that the arguments preceding Claim 7 use only the conclusion of Theorem 4.9. Thus we can repeat the arguments and see that the outer boundary layer, $J^{\prime}$, of $M^{\prime}$ also satisfies the conclusion of Theorem 4.11. If $J \cup J^{\prime}=M$, then we see from Claim 7 that $M$ is as described in Theorem 4.11. If $J \cup J^{\prime} \varsubsetneqq M$, we can repeat these arguments, and obtain Theorem 4.11. 


\section{Proof of the only if Part of Main Theorem 2.7}

We first note that the special case where $p=2$ can be settled very easily as follows. In this case, $K(1 / 2)$ is the Hopf link, and $G(K(1 / 2))=\left\langle a, b \mid u_{1 / 2}\right\rangle$ with $u_{1 / 2}=a b a^{-1} b^{-1}$ is the rank 2 free abelian group with basis $\{a, b\}$. On the other hand, $I_{1}(1 / 2) \cup I_{2}(1 / 2)=\{0,1\}$, and it is obvious that $u_{0}=a b$ is not conjugate to $u_{1}^{ \pm 1}=\left(a b^{-1}\right)^{ \pm 1}$ in $G(K(1 / 2))$, so that $\alpha_{0}$ is not homotopic to $\alpha_{1}$ in $S^{3}-K(1 / 2)$. This implies that the unoriented simple loops $\alpha_{s}$ fall into precisely three homotopy classes (represented by $\alpha_{0}, \alpha_{1}$ and $\alpha_{\infty}$, the last one trivial). Hence the only if part of Main Theorem 2.7 is valid when $p=2$.

Proof. of the only if part of Main Theorem 2.7 As noted in the beginning of this section, we may assume that $p$ is a positive integer with $p \geq 3$. For two distinct elements $s, s^{\prime} \in I_{1}(1 / p) \cup I_{2}(1 / p)=\{0\} \cup\left[\frac{1}{p-1}, 1\right]$, suppose that the unoriented loops $\alpha_{s}$ and $\alpha_{s^{\prime}}$ are homotopic in $S^{3}-K(1 / p)$. Then $u_{s}$ and $u_{s^{\prime}}^{ \pm 1}$ are conjugate in $G(K(1 / p))$. Let $R$ be the symmetrized subset of $F(a, b)$ generated by the single relator $u_{1 / p}$ of the upper presentation of $G(K(1 / p))$. Due to Lemma 4.7, there is a reduced nontrivial annular $R$-diagram $M$ such that $u_{s}$ and $u_{s^{\prime}}^{ \pm 1}$ are, respectively, outer and inner boundary labels of $M$. Then we see that $M$ satisfies the three hypotheses (i), (ii) and (iii) of Theorem 4.9. In fact, hypothesis (iii) follows from Proposition 3.19)(1) and Remark 3.20.

Lemma 5.1. Suppose that $\left(\left(b_{1}, b_{2}, \ldots, b_{n}\right)\right)$ denotes the cyclic $S$-sequence of an outer boundary label of $M$. Then $b_{i}<p$ and the cyclic $S$-sequence of an inner boundary label of $M$ is $\left(\left(p-b_{1}, p-b_{2}, \ldots, p-b_{n}\right)\right)$.

We complete the proof of the only if part of Main Theorem 2.7, by assuming the above lemma. Note that the cyclic $S$-sequences of arbitrary outer and inner boundary labels of $M$ are $C S\left(u_{s}\right)$ and $C S\left(u_{s^{\prime}}^{ \pm 1}\right)=C S\left(u_{s^{\prime}}\right)$, respectively. Putting $C S\left(u_{s}\right)=\left(\left(b_{1}, b_{2}, \ldots, b_{n}\right)\right)$, Lemma 5.1 implies that $C S\left(u_{s^{\prime}}\right)=((p-$ $\left.\left.b_{1}, p-b_{2}, \ldots, p-b_{n}\right)\right)$. In particular, $C S\left(u_{s}\right)$ and $C S\left(u_{s^{\prime}}\right)$ have the same length. Since the length of $C S\left(u_{s}\right)$ is even or 1 according as $s \neq 0$ or $s=0$ (see Lemma 3.6(1) and Remark 3.7) and since $s$ and $s^{\prime}$ are distinct, we see that $s$ nor $s^{\prime}$ is 0 . Hence we may write $s=q_{1} / p_{1}$ and $s^{\prime}=q_{2} / p_{2}$, where $\left(p_{i}, q_{i}\right)$ is a pair of relatively prime positive integers. Then by Lemma 3.6(1), we have $n=2 q_{1}=2 q_{2}$, so $q_{1}=q_{2}$. Also by Lemma 3.6(1),

$$
\sum_{i=1}^{q_{1}} b_{i}=\sum_{i=1}^{q_{1}} \nu_{i}(s)=\left\lfloor p_{1}\right\rfloor_{*}-\lfloor 0\rfloor_{*}=p_{1},
$$


where $\nu_{i}(s)$ denotes the $i$-th term of $S\left(u_{s}\right)$, and similarly

$$
\sum_{i=1}^{q_{1}}\left(p-b_{i}\right)=\sum_{i=1}^{q_{1}} \nu_{i}\left(s^{\prime}\right)=\left\lfloor p_{2}\right\rfloor_{*}-\lfloor 0\rfloor_{*}=p_{2},
$$

where $\nu_{i}\left(s^{\prime}\right)$ denotes the $i$-th term of $S\left(u_{s^{\prime}}\right)$. Hence $p_{2}=\sum_{i=1}^{q_{1}}\left(p-b_{i}\right)=p q_{1}-p_{1}$, which implies that $q_{1} /\left(p_{1}+p_{2}\right)=1 / p$, as required.

It remains to prove Lemma 5.1.

Proof. of Lemma 5.1 Let $J$ be the outer boundary layer of $M$. Due to Theorem 4.11, $J$ is as depicted in Figure 7(a). Let $\alpha$ and $\delta$ be, respectively, the outer and inner boundary cycles of $J$ starting from $v_{0}$, where $v_{0}$ is a vertex lying in both the outer and inner boundaries of $J$. Here, recall from Convention 4.6 that $\alpha$ is read clockwise and $\delta$ is read counterclockwise. Let $\alpha=e_{1}, e_{2}, \ldots, e_{2 m}$ and $\delta^{-1}=e_{1}^{\prime}, e_{2}^{\prime}, \ldots, e_{2 m}^{\prime}$ be the decompositions into oriented edges in $\partial J$. Then clearly for each $j=1, \ldots, m$, there is a face $D_{j}$ of $J$ such that $e_{2 j-1}, e_{2 j}, e_{2 j}^{\prime-1}, e_{2 j-1}^{\prime-1}$ are consecutive edges in a boundary cycle of $D_{j}$.

Claim 1. For each 2-cell $D_{j}$, there are decompositions $v_{2} \equiv v_{2 b} v_{2 e}$ and $v_{4} \equiv$ $v_{4 b} v_{4 e}$ such that the following hold, where $u_{1 / p} \equiv v_{2} v_{4}$ as in Lemma 3.24, and $v_{i b}$ and $v_{i e}$ are nonempty initial and terminal subwords of $v_{i}$.

(1) If $\left(\phi\left(\partial D_{j}\right)\right) \equiv\left(u_{1 / p}\right)$, then $\left(\phi\left(e_{2 j-1}\right), \phi\left(e_{2 j}\right), \phi\left(e_{2 j}^{\prime-1}\right), \phi\left(e_{2 j-1}^{\prime-1}\right)\right)$ is equal to $\left(v_{2 e}, v_{4 b}, v_{4 e}, v_{2 b}\right)$ or $\left(v_{4 e}, v_{2 b}, v_{2 e}, v_{4 b}\right)$.

(2) If $\left(\phi\left(\partial D_{j}\right)\right) \equiv\left(u_{1 / p}^{-1}\right)$, then $\left(\phi\left(e_{2 j}^{-1}\right), \phi\left(e_{2 j-1}^{-1}\right), \phi\left(e_{2 j-1}^{\prime}\right), \phi\left(e_{2 j}^{\prime}\right)\right)$ is equal to $\left(v_{2 e}, v_{4 b}, v_{4 e}, v_{2 b}\right)$ or $\left(v_{4 e}, v_{2 b}, v_{2 e}, v_{4 b}\right)$.

Proof. of Claim 1 We treat the case where $\left(\phi\left(\partial D_{j}\right)\right) \equiv\left(u_{1 / p}\right)$. (The other case can be treated similarly.) Since $\phi\left(e_{2 j-1}\right) \phi\left(e_{2 j}\right)$ is not a piece by Convention 4.3, it contains a maximal 1-piece as a proper initial subword, $v$. Assume that $v$ is $v_{2 b}^{*}$ (resp., $\left.v_{4 b}^{*}\right)$ (see Lemma 3.24(1a)). Since $\phi\left(e_{2 j-1}\right) \phi\left(e_{2 j}\right)$ is contained in a maximal 2-piece, this assumption together with Lemma 3.24(1b) implies that $\phi\left(e_{2 j-1}\right) \phi\left(e_{2 j}\right)$ is $v_{2}$ or $v_{4}$, and hence $S\left(\phi\left(e_{2 j-1}\right) \phi\left(e_{2 j}\right)\right)=(p)$. But, since $s \in$ $I_{1}(1 / p) \cup I_{2}(1 / p)=\{0\} \cup\left[\frac{1}{p-1}, 1\right]$, every term of the cyclic word $\left(u_{s}\right)=(\phi(\alpha))$ is at most $p-1$ by Proposition 3.19 (2) and by Remark 3.20. This implies that the cyclic word $\left(u_{s}\right)=(\phi(\alpha))$ cannot contain a subword whose $S$-sequence is $(p)$, a contradiction. Hence, the word $v$ is equal to $v_{2 e}$ or $v_{4 e}$ by Lemma 3.24)(1a). This together with Lemma 3.24 implies that $\left(\phi\left(e_{2 j-1}\right), \phi\left(e_{2 j}\right)\right)=\left(v_{2 e}, v_{4 b}\right)$ or $\left(v_{4 e}, v_{2 b}\right)$ accordingly. By applying a parallel argument to $\left(\phi\left(e_{2 j}^{\prime-1}\right), \phi\left(e_{2 j-1}^{\prime-1}\right)\right)$ 
and by using the fact that the cyclic word $\left(\phi\left(\partial D_{j}\right)\right)$ is equal to $\left(u_{1 / p}\right)$, we obtain the desired result.

By Claim 1, there are positive integers $\ell_{i}$ and $\ell_{i}^{\prime}$, for each $1 \leq i \leq 2 m$, such that $S\left(\phi\left(e_{i}\right)\right)=\left(\ell_{i}\right)$ and $S\left(\phi\left(e_{i}^{\prime}\right)\right)=\left(\ell_{i}^{\prime}\right)$ and such that $\ell_{i}+\ell_{i}^{\prime}=p$. Furthermore, they satisfy the following.

Claim 2. For each $1 \leq j \leq m$, the following hold.

(1) $S\left(\phi\left(e_{2 j-1}\right) \phi\left(e_{2 j}\right)\right)=\left(\ell_{2 j-1}, \ell_{2 j}\right)$ and $S\left(\phi\left(e_{2 j-1}^{\prime}\right) \phi\left(e_{2 j}^{\prime}\right)\right)=\left(\ell_{2 j-1}^{\prime}, \ell_{2 j}^{\prime}\right)$.

(2) $S\left(\phi\left(e_{2 j}\right) \phi\left(e_{2 j+1}\right)\right)=\left(\ell_{2 j}, \ell_{2 j+1}\right)$ and $S\left(\phi\left(e_{2 j}^{\prime}\right) \phi\left(e_{2 j+1}^{\prime}\right)\right)=\left(\ell_{2 j}^{\prime}, \ell_{2 j+1}^{\prime}\right)$, where the indices are taken modulo $2 \mathrm{~m}$.

Proof. of Claim 2 The first assertion immediately follows from Claim 1. To see the second assertion, note that Claim 1 implies that if $\phi\left(e_{2 j-1}\right)$ is a positive (resp., negative) word then $\phi\left(e_{2 j}^{\prime}\right)$ is also a positive (resp., negative) word and $\phi\left(e_{2 j-1}^{\prime}\right)$ and $\phi\left(e_{2 j}\right)$ are negative (resp., positive) words. In particular, if $\phi\left(e_{i}\right)$ is a positive (resp., negative) word then $\phi\left(e_{i}^{\prime}\right)$ is a negative (resp., positive) word, i.e., $\phi\left(e_{i}\right)$ and $\phi\left(e_{i}^{\prime}\right)$ always have "opposite signs". Now consider the $S$-sequence of $\phi\left(e_{2 j}\right) \phi\left(e_{2 j+1}\right)$. Clearly $S\left(\phi\left(e_{2 j}\right) \phi\left(e_{2 j+1}\right)\right)$ is either $\left(\ell_{2 j}+\ell_{2 j+1}\right)$ or $\left(\ell_{2 j}, \ell_{2 j+1}\right)$. We will show that only the latter is possible.

Suppose on the contrary that $S\left(\phi\left(e_{2 j}\right) \phi\left(e_{2 j+1}\right)\right)$ is $\left(\ell_{2 j}+\ell_{2 j+1}\right)$ for some $j$. Then $\phi\left(e_{2 j}\right)$ and $\phi\left(e_{2 j+1}\right)$ share the same sign and hence $\phi\left(e_{2 j}^{\prime}\right)$ and $\phi\left(e_{2 j+1}^{\prime}\right)$ share the same sign by the above observation. So $S\left(\phi\left(e_{2 j}^{\prime}\right) \phi\left(e_{2 j+1}^{\prime}\right)\right)$ is $\left(\ell_{2 j}^{\prime}+\right.$ $\left.\ell_{2 j+1}^{\prime}\right)$. By Proposition [3.19(2) and Remark 3.20, $\left(u_{s}\right)=(\phi(\alpha))$ cannot contain a subword whose $S$-sequence is $(p)$. So $\ell_{2 j}+\ell_{2 j+1}<p$. But then $S\left(\phi\left(e_{2 j}^{\prime}\right) \phi\left(e_{2 j+1}^{\prime}\right)\right)$ is $\left(\ell_{2 j}^{\prime}+\ell_{2 j+1}^{\prime}\right)=\left(2 p-\ell_{2 j}-\ell_{2 j+1}\right)$ with $2 p-\ell_{2 j}-\ell_{2 j+1}>p$. If $J=M$, this contradicts the assumption that $s^{\prime} \in I_{1}(1 / p) \cup I_{2}(1 / p)=$ $\{0\} \cup\left[\frac{1}{p-1}, 1\right]$, because every term of $C S\left(s^{\prime}\right)=C S\left(u_{s^{\prime}}\right)$ has to be at most $p-1$ again by Proposition [3.19(2) and Remark 3.20. Also if $J \subsetneq M$, then, as depicted in Figure $7(\mathrm{~b}), e_{2 j}^{\prime}$ and $e_{2 j+1}^{\prime}$ are two consecutive edges in $\partial D_{j}^{\prime} \cap \delta^{-1}$ (recall that $\delta$ is the inner boundary cycle of $J$ starting from $v_{0}$ and is read counterclockwise) for some face $D_{j}^{\prime}$ in $M-J$, but then $\left(u_{1 / p}\right)$ contains a subword whose $S$-sequence is $\left(2 p-\ell_{2 j}-\ell_{2 j+1}\right)$ with $2 p-\ell_{2 j}-\ell_{2 j+1}>p$, contradicting $C S\left(u_{1 / p}\right)=((p, p))$.

Therefore $C S(\phi(\alpha))$ becomes $\left(\left(\ell_{1}, \ell_{2}, \ldots, \ell_{2 m-1}, \ell_{2 m}\right)\right)$, and $C S\left(\phi\left(\delta^{-1}\right)\right)$ becomes $\left(\left(p-\ell_{1}, p-\ell_{2}, \ldots, p-\ell_{2 m-1}, p-\ell_{2 m}\right)\right)$. If $M=J$, the proof of Lemma 5.1 is completed. Now assume $J \subsetneq M$. Let $J_{1}$ denote the outer boundary layer of $M-\operatorname{int}(J)$. Then the cyclic $S$-sequence of an arbitrary inner boundary label of $J_{1}$ is $\left(\left(\ell_{1}, \ell_{2}, \ldots, \ell_{2 m-1}, \ell_{2 m}\right)\right)$. If $M=J \cup J_{1}$, then $s=s^{\prime}$, a contradiction. 
Hence we must have $J \cup J_{1} \subsetneq M$. Let $J_{2}$ denote the outer boundary layer of $M-\operatorname{int}\left(J \cup J_{1}\right)$. Then the cyclic $S$-sequence of an arbitrary inner boundary label of $J_{2}$ is $\left(\left(p-\ell_{1}, p-\ell_{2}, \ldots, p-\ell_{2 m-1}, p-\ell_{2 m}\right)\right)$. If $M=J \cup J_{1} \cup J_{2}$, the proof of Lemma 5.1 is completed. By the repetition of this argument, we are done.

\section{ACKNOWLEDGEMENT}

The authors would like to thank Koji Fujiwara for his interest in this work and for inspiring discussion. They would also like to thank the referee for very careful reading and valuable comments and suggestions, especially on the

proof of the if part of the main theorem. Their thanks also go to the editor of this journal for kindly and properly handling this paper and its two sequels.

\section{REFERENCES}

[1] Appel, K. I., Schupp, P. E.: The conjugacy problem for the group of any tame alternating knot is solvable. Proc. Amer. Math. Soc. 33, 329-336 (1972)

[2] Crowell, R. H., Fox, R. H.: Introduction to knot theory. Reprint of the 1963 original. Graduate Texts in Math. 57, Springer-Verlag, New York-Heidelberg (1977)

[3] Johnsgard, K.: The conjugacy problem for the groups of alternating prime tame links is polynomial-time. Trans. Amer. Math. Soc. 349, 857-901 (1977)

[4] Komori, Y., Series, C.: The Riley slice revised. In the Epstein Birthday Shrift. I. Rivin, C. Rourke and C. Series eds. Geom. Topol. Monogr. 1, 303-316 (1999)

[5] Lee, D., Sakuma, M.: Simple loops on 2-bridge spheres in 2-bridge link complements. Electron. Res. Announc. Math. Sci. 18, 97-111 (2011)

[6] Lee, D., Sakuma, M.: Epimorphisms between 2-bridge link groups: homotopically trivial simple loops on 2-bridge spheres. Proc. London Math. Soc. 104, 359-386 (2012)

[7] Lee, D., Sakuma, M.: Homotopically equivalent simple loops on 2-bridge spheres in 2-bridge link complements (II). Geom. Dedicata (to appear). arXiv:1103.0856

[8] Lee, D., Sakuma, M.: Homotopically equivalent simple loops on 2-bridge spheres in 2-bridge link complements (III). Geom. Dedicata (to appear). arXiv:1111.3562

[9] Lee, D., Sakuma, M.: A variation of McShane's identity for 2-bridge links. Geom. Topol. (to appear). arXiv:1112.5859

[10] Lyndon, R. C., Schupp, P. E.: Combinatorial group theory. Springer-Verlag, Berlin (1977)

[11] Ohtsuki, T., Riley, R., Sakuma, M.: Epimorphisms between 2-bridge link groups. Geom. Topol. Monogr. 14, 417-450 (2008)

[12] Préaux, J. P.: Conjugacy problems in groups of oriented geometrizable 3-manifolds. Topology 45, 171-208 (2006)

[13] Ratcliffe, J. G.: Foundations of hyperbolic manifolds. Graduate Texts in Math. 149, Springer-Verlag, New York (1994) 
[14] Riley, R.: Parabolic representations of knot groups, I. Proc. London Math. Soc. 24, 217-242 (1972)

[15] Sela, Z.: The conjugacy problem for knot groups. Topology 32, 363-369 (1993)

[16] Weinbaum, C. M.: The word and conjugacy problems for the knot group of any tame, prime, alternating knot. Proc. Amer. Math. Soc. 30, 22-26 (1971)

Department of Mathematics, Pusan National University, San-30 JangjeonDong, Geumuung-Gu, Pusan, 609-735, Republic of Korea

E-mail address: donghi@pusan.ac.kr

Department of Mathematics, Graduate School of Science, Hiroshima University, Higashi-Hiroshima, 739-8526, Japan

E-mail address: sakuma@math.sci.hiroshima-u.ac.jp 